\title{
FIRST COSMOLOGY RESULTS USING TYPE Ia SUPERNOVAE FROM THE DARK ENERGY SURVEY: EFFECTS OF CHROMATIC CORRECTIONS TO SUPERNOVA PHOTOMETRY ON MEASUREMENTS OF COSMOLOGICAL PARAMETERS
}

J. Lasker ${ }^{1,2 \star}$, R. Kessler ${ }^{1,2}$, D. Scolnic ${ }^{2}$, D. Brout ${ }^{3}$, D. L. Burke ${ }^{4,5}$, C. B. D’Andrea ${ }^{3}$, T. M. Davis ${ }^{6}$, S. R. Hinton ${ }^{6}$, A. G. Kim ${ }^{7}$, T. S. Li ${ }^{8,2}$, C. Lidman ${ }^{9}$, E. Macaulay ${ }^{10}$, A. Möller ${ }^{11,9}$, E. S. Rykoff ${ }^{4,5}$, M. Sako ${ }^{3}$, M. Smith ${ }^{12}$, M. Sullivan ${ }^{12}$, E. Swann ${ }^{10}$, B. E. Tucker ${ }^{11,9}$, W. Wester ${ }^{8}$, B. A. Bassett ${ }^{13,14}$, T. M. C. Abbott ${ }^{15}$, S. Allam ${ }^{8}$, J. Annis ${ }^{8}$, S. Avila ${ }^{10}$, K. Bechtol ${ }^{16}$, E. Bertin ${ }^{17,18}$, D. Brooks ${ }^{19}$, A. Carnero Rosell ${ }^{20,21}$, M. Carrasco Kind ${ }^{22,23}$, J. Carretero ${ }^{24}$, F. J. Castander ${ }^{25,26}$, J. Calcino ${ }^{6}$, D. Carollo ${ }^{27}$, L. N. da Costa ${ }^{21,28}$, C. Davis ${ }^{4}$, J. De Vicente ${ }^{20}$, H. T. Diehl ${ }^{8}$, P. Doel ${ }^{19}$, A. Drlica-Wagner ${ }^{8,2}$, B. Flaugher ${ }^{8}$, J. Frieman ${ }^{8,2}$, J. García-Bellido ${ }^{29}$, E. Gaztanaga ${ }^{25,26}$, D. Gruen ${ }^{4,5}$, R. A. Gruendl ${ }^{22,23}$, J. Gschwend ${ }^{21,28}$, G. Gutierrez ${ }^{8}$, D. L. Hollowood ${ }^{30}$, K. Honscheid ${ }^{31,32}$, J. K. Hoormann ${ }^{6}$, D. J. James ${ }^{33}$, S. Kent ${ }^{8,2}$, E. Krause ${ }^{34}$,

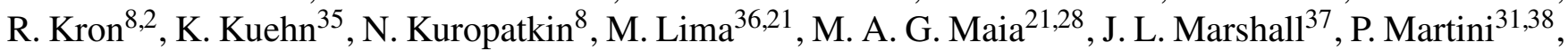
F. Menanteau ${ }^{22,23}$, C. J. Miller ${ }^{39,40}$, R. Miquel ${ }^{41,24}$, A. A. Plazas ${ }^{42}$, E. Sanchez ${ }^{20}$, V. Scarpine ${ }^{8}$, I. SevillaNoarbe $^{20}$, R. C. Smith ${ }^{15}$, M. Soares-Santos ${ }^{43}$, F. Sobreira ${ }^{44,21}$, E. Suchyta ${ }^{45}$, M. E. C. Swanson ${ }^{23}$, G. Tarle ${ }^{40}$, D. L. Tucker ${ }^{8}$, A. R. Walker ${ }^{15}$

(DES Collaboration)

Affiliations are in Appendix A.

Last updated 2015 May 22; in original form 2013 September 5

\begin{abstract}
Calibration uncertainties have been the leading systematic uncertainty in recent analyses using type Ia Supernovae (SNe Ia) to measure cosmological parameters. To improve the calibration, we present the application of Spectral Energy Distribution (SED)-dependent "chromatic corrections" to the supernova light-curve photometry from the Dark Energy Survey (DES). These corrections depend on the combined atmospheric and instrumental transmission function for each exposure, and they affect photometry at the $0.01 \mathrm{mag}(1 \%)$ level, comparable to systematic uncertainties in calibration and photometry. Fitting our combined DES and low- $z$ SN Ia sample with Baryon Acoustic Oscillation (BAO) and Cosmic Microwave Background (CMB) priors for the cosmological parameters $\Omega_{\mathrm{m}}$ (the fraction of the critical density of the universe comprised of matter) and $w$ (the dark energy equation of state parameter), we compare those parameters before and after applying the corrections. We find the change in $w$ and $\Omega_{\mathrm{m}}$ due to not including chromatic corrections are -0.002 and 0.000, respectively, for the DES-SN3YR sample with BAO and CMB priors, consistent with a larger DESSN3YR-like simulation, which has a $w$-change of 0.0005 with an uncertainty of 0.008 and an $\Omega_{\mathrm{m}}$ change of 0.000 with an uncertainty of 0.002 . However, when considering samples on individual CCDs we find large redshift-dependent biases ( $\sim 0.02$ in distance modulus) for supernova distances.
\end{abstract}

Key words: cosmology:dark energy-cosmology:observations-supernovae:generaltechniques: photometric 


\section{Lasker et al.}

shifts, which, when combined with a measurement of the redshift, are used to trace the expansion history of the Universe. The SN Ia distances and redshifts are fit to a model that is typically parametrized in terms of the fraction of the universe's energy that is in matter $\left(\Omega_{\mathrm{M}}\right)$ versus that which is in dark energy $\left(\Omega_{\Lambda}\right)$, as well as the equation of state parameter of dark energy, $w$.

The recovery of cosmological parameters from $\mathrm{SNe}$ is sensitive to calibration in two ways. First, cosmological constraints depend on comparing the relative brightnesses of SNe at different redshifts. As the rest frame SN spectrum is redshifted, we observe it in different bandpasses which must be calibrated relative to each other. Second, we observe SNe at different positions on the sky, different locations on our focal plane, and in different weather conditions. Non-uniformity of these observations can introduce potential cosmological biases. Together, these calibration uncertainties make up the largest source of systematic uncertainty on cosmological parameters derived from SN Ia distances.

The impact of the systematic uncertainty from calibration is well illustrated in the recent analysis of the Pantheon sample (Scolnic et al. 2017), which is the largest combined sample of spectroscopically confirmed SNe Ia analyzed to date. For the dark energy equation of state parameter $w$, the Pantheon analysis' calibration uncertainty of 2-6 mmag, depending on sample, contributes $\sigma_{w}=0.02$, half of their total uncertainty on $w$.

The samples included in this analysis are from the PanSTARRS 1 (PS1, Rest et al. 2014) Medium Deep Survey, the Sloan Digital Sky Survey II (SDSS-II, Sako et al. 2014), the Supernova Legacy Survey (SNLS, Conley et al. 2011), HST (Riess et al. 2004, 2007; Rodney et al. 2014), the Center for Astrophysics low redshift surveys (CFA3 and CFA4, Hicken et al. 2009 and Hicken et al. 2012), the Carnegie Supernova Project (CSP, Stritzinger et al. 2011), and the HST Cluster Supernova Survey (Suzuki et al. 2012).

It's critical to note that while the calibration uncertainties from these samples are a factor of 50 below the distance uncertainties, the binned distance uncertainties that constrain cosmology are reduced as $\left(\sqrt{N_{\mathrm{SN}}}\right)$, unlike the calibration error.

It is important to reduce calibration uncertainties in order to utilise the improved statistical power in measuring cosmological parameters from surveys with larger samples. The Dark Energy Survey Supernova Program (DES-SN, Kessler et al. 2015) is measuring multi-band light curves of a photometric sample of thousands of SNe Ia, as well as a spectroscopically classified sample of several hundred SNe Ia. Furthermore, the Large Synoptic Survey Telescope (LSST, Ivezić \& the LSST Science Collaboration 2013), which is expected to begin survey operations in 2022, will discover $10^{4} \mathrm{SNe}$ Ia with high-quality light curves in its deep-drilling fields, as well as over a million SNe Ia with sparser light curves in the wide-fast-deep survey.

Calibration of astronomical images is fundamentally the transformation of a number of ADU (Analog/Digital Units) from a source in a CCD image to a top-of-the-atmosphere brightness. This process has undergone many different iterations throughout the last 20 years of wide area astrophysical sky surveys. We briefly summarise these below.

The Sloan Digital Sky Survey (SDSS, York et al. 2000) made many innovations for the calibration procedures of wide-area sky surveys. They developed the ugriz filter system (Fukugita et al. 1996) that has been used with minor variations by many other surveys, including DES (Flaugher et al. 2015), PS1 (Tonry et al. 2012) and SNLS (Regnault et al. 2009). The Ubercal method (Padmanabhan et al. 2008) accounted for the flat field variation and amplifier gain variation while absorbing the atmospheric effects into a linear (in magnitude) airmass correction. This method made use of repeated observations of stars during the survey to achieve $1 \%$ relative calibration (consistency in the natural magnitude system) across the survey footprint. Since Vega was too bright to be observed by SDSS, they tied their absolute photometry to the AB system (Oke \& Gunn 1983), a hypothetical flat reference spectrum which has a constant value of $3631 \mathrm{Jy}\left(1 \mathrm{Jy}=10^{-26} \frac{\mathrm{W}}{\mathrm{m}^{2} \mathrm{~Hz}}\right)$ as would be measured at the top of the atmosphere. The $\mathrm{AB}$ system provides a more practical path to apply the absolute calibration through observations of fainter flux standards like BD+17-4708, which can be observed by large survey instruments without saturating the CCDs.

PS1 improved on the Ubercal method that SDSS used for its relative calibration by adopting a different survey strategy (Magnier et al. 2013). This included larger areas of overlap between exposures and spacing repeat observations of a field both on 15 minute timescales within a night and at 6 month separations. These overlaps enabled PS1 to obtain high-quality calibration on nights with poor conditions as explained in Schlafly et al. (2012). PS1 used their improved photometry and overlaps of their fields with those of SDSS to recalibrate SDSS to PS1-levels of precision using a method called Hypercalibration (Finkbeiner et al. 2016).

To further improve the absolute calibration, PS1 measured the full transmission function including instrument (telescope + CCD) and atmosphere. They measured the instrumental transmission using in-dome monochromator scans of the telescope and CCDs without filters, and they also utilised the vendor scans of the filter throughput (Tonry et al. 2012). For the atmospheric component, they included MODerate resolution atmospheric TRANsmission (MODTRAN, Berk et al. 1987) models in their method to allow for specific contributions from aerosols, water vapor, and ozone to the linear airmass extinction. PS1 used repeated observations of many HST CalSpec (Bohlin et al. 2014) standard stars inside of the footprint to tie its photometry to the $\mathrm{AB}$ system (Scolnic et al. 2015). Hereafter "transmission" refers to the full instrumental + atmospheric transmission function unless otherwise specified.

For the Dark Energy Survey (The Dark Energy Survey Collaboration 2005) at the Cerro Tololo Interamerican Observatory (CTIO), a new calibration method has been developed based on a forward modeling approach. This method is called the Forward Global Calibration Method (FGCM, Burke et al. 2018, B18 hereafter). While SDSS and PS1 account for the effect of the atmosphere averaged over each night, FGCM models the full DES transmission function for each CCD and each exposure, thus accounting for dependence of the transmission function on focal plane position as well as its time variation. FGCM uses approximately bimonthly measurements of the system throughput in each passband for each CCD (DECals, Marshall et al. 2013). More information about the variation in the instrumental transmission function across the focal plane is obtained from star flats as described in Drlica-Wagner et al. 2018. To monitor atmospheric changes, FGCM uses data from a GPS receiver at CTIO (Blake \& Shaw 2011; Flaugher et al. 2015). The data is analyzed by SuomiNet ${ }^{1}$, which provides measurements of atmospheric precipitable water vapor (PWV) in 30 minute time windows. It uses this information in conjunction with data from bright stars in normal DES observations. FGCM achieves relative calibration at the $\sim 4.5 \mathrm{mmag}$ level. This is based on a comparison

\footnotetext{
1 http://www.suominet.ucar.edu
} 
of the DES catalogs to those of Gaia DR2 averaged over small patches of sky and is likely an upper bound for the uncertainty.

FGCM determines its absolute calibration by comparing observed magnitudes of the CalSpec standard C26202 with the "synthetic" magnitudes obtained by multiplying the CalSpec spectrum with each filter's model FGCM transmission function. C26202 is located within one of the DES-SN deep fields that has been observed over 100 times during the survey and it is faint enough to not saturate in most of the exposures. The flux scaling for each exposure, usually expressed as the logarithmic zeropoint, is obtained by integrating the product of the exposure's transmission function with a reference spectrum. Despite using C26202 to determine the absolute calibration, DES uses the flat $\mathrm{AB}$ spectrum as its reference.

This zeropoint is precisely correct only if the source SED is the reference spectrum or if it is observed under the exact conditions that define the reference transmission functions. The reference transmission functions are chosen during the FGCM fitting process to represent the average DES transmission function in each band. In order to take observations of other sources in other observing conditions and place them on the system defined by the reference transmission function, the optimal calibration requires additional corrections that depend on the SED of the source being observed. We call these "chromatic corrections."

There are two observational effects that contribute to the need for chromatic corrections. First, the atmospheric transmission as a function of wavelength varies between observations. Those variations are illustrated in the uppermost panel of Fig. 3 from Li et al. (2016) (hereafter, L16). This figure shows the ratio between the transmission functions at PWV $=3 \mathrm{~mm}$ and $\mathrm{PWV}=10 \mathrm{~mm}$. This plot shows a maximum of $50 \%$ fractional variation in the transmission function in $z$-band due to the PWV variation. Second, the Dark Energy Camera (DECam Flaugher et al. 2015) filter transmission function varies across the focal plane as shown in Fig. 6 of L16. This figure shows a shift of the edge of the $i$-band transmission function of up to $6 \mathrm{~nm}$ as a function of distance from the centre of the focal plane.

Color differences between astrophysical point sources and the reference standard affect the size of these chromatic corrections. This is particularly important for supernovae, as supernova SEDs are much redder than the reference standard, are very diverse, have strong broad features, vary with time, and vary significantly in color due to the wide range of redshifts observed as well reddening due to dust in the SN host galaxy. The variation of SN Ia spectra with redshift is shown here in Fig. 1. The objective of this paper is to demonstrate the application of the chromatic corrections to DES$\mathrm{SN}$ data and characterise the effects of the corrections in singleepoch photometry, light curves, and cosmology.

The outline of the paper is as follows. The formalism of chromatic corrections is described in $\$ 2.1$. We describe the dataset to which these SED-based corrections are applied in $\$ 2.2$. We show the method with which the SN Ia light curves are fit, including the application of the chromatic corrections, in $\$ 2.3$. In $\$ 3$, we show our results including: demonstrating the effect of the corrections on the single-epoch photometry $(\$ 3.1)$; the effect on the nuisance parameters $\alpha$ and $\beta$ as well as a cross-check of those parameters with those of Pantheon in $\$ 3.2$; the effect on the supernova light curves and cosmology (\$3.3); and the effect of the corrections on SN Ia simulated on individual CCDs (\$3.4). Finally we examine several cross checks on our analysis in $\$ 3.5$. $[\mathrm{h} !]$
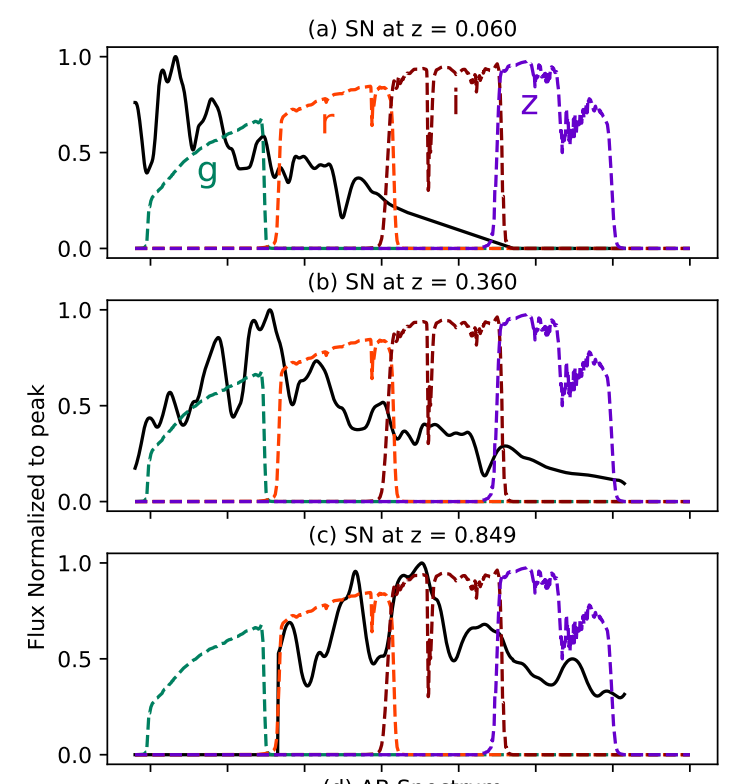

(d) AB Spectrum

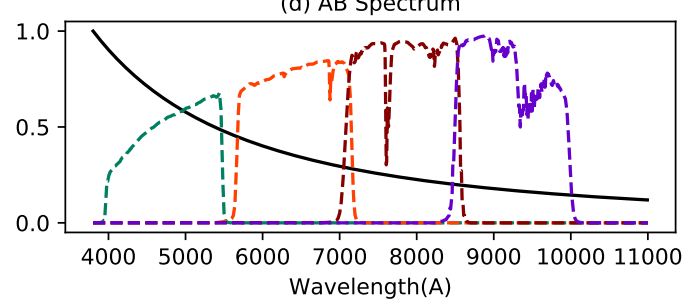

Figure 1. SN Ia SEDs at peak brightness for (a) low, (b) intermediate, and (c) high redshift model spectra from SALT2 Guy et al. 2007, and (d) the $\mathrm{AB}$ spectrum, which is proportional to $1 / \lambda^{2}$. Overplotted on all spectra are the DES griz standard bandpasses.

\section{METHODS + DATA SAMPLE}

\subsection{Application of chromatic corrections}

Here we describe the exact form of the chromatic corrections and the manner in which they are applied to the supernova photometry.

The typical definition of the magnitude, $m_{b}$, in a band, $b$, of a source with flux (photon counts normalised by telescope aperture and exposure time), $F_{b}$, in an image with zeropoint, $Z P_{b}$, is

$m_{b}=-2.5 \log _{10}\left(F_{b}\right)+Z P_{b}$.

In the $\mathrm{AB}$ system, this definition can be further expanded such that:

$$
\begin{aligned}
m_{b}= & -2.5 \log _{10} \int_{0}^{\infty} F_{\nu, \operatorname{src}}(\lambda) \phi_{\mathrm{b}, \text { tot }}(\lambda) \lambda^{-1} d \lambda \\
& +2.5 \log _{10} \int_{0}^{\infty} F_{\nu, \text { ref }}(\lambda) \phi_{\mathrm{b}, \text { tot }}(\lambda) \lambda^{-1} d \lambda,
\end{aligned}
$$

where $F_{v, \operatorname{src}}(\lambda)$ is the SED (in units of $\frac{\mathrm{W}}{\mathrm{m}^{2} \mathrm{~Hz}}$ ) of the source object being observed, and $F_{v \text {,ref }}(\lambda)$ is the $\mathrm{SED}$ of the reference ob- 
ject for the photometric system. For DES, we use the AB spectrum (Fig. 1d). $\phi_{\mathrm{b}, \text { tot }}(\lambda)$ is the dimensionless total transmission function. This definition of the magnitude forms the basis for the chromatic corrections in L16:

$$
\begin{aligned}
\delta_{b}^{m} & =m_{\mathrm{std}}-m_{\mathrm{obs}}= \\
& -2.5 \log _{10} \frac{\int_{0}^{\infty} F_{v, \mathrm{src}}(\lambda) \phi_{\mathrm{obs}}^{\mathrm{atm}}(\lambda) \phi_{\mathrm{b}, \mathrm{obs}}^{\mathrm{inst}}(\lambda) \lambda^{-1} d \lambda}{\int_{0}^{\infty} F_{\nu, \mathrm{src}}(\lambda) \phi_{\mathrm{ref}}^{\mathrm{atm}}(\lambda) \phi_{\mathrm{b}, \mathrm{ref}}^{\mathrm{inst}}(\lambda) \lambda^{-1} d \lambda} \\
& +2.5 \log _{10} \frac{\int_{0}^{\infty} F_{v, \mathrm{ref}}(\lambda) \phi_{\mathrm{obs}}^{\mathrm{atm}}(\lambda) \phi_{\mathrm{b}, \mathrm{obs}}^{\mathrm{inst}}(\lambda) \lambda^{-1} d \lambda}{\int_{0}^{\infty} F_{v, \mathrm{ref}}(\lambda) \phi_{\mathrm{ref}}^{\mathrm{atm}}(\lambda) \phi_{\mathrm{b}, \mathrm{ref}}^{\text {inst }}(\lambda) \lambda^{-1} d \lambda} .
\end{aligned}
$$

In Eq. 3, $m_{\text {std }}$ is the "standard" magnitude of the object being observe transformed as though it was observed under the reference conditions, $m_{\mathrm{obs}}$ is the magnitude that was observed under the actual conditions, $F_{v \text {, src }}$ and $F_{v \text {,ref }}$ are the same as in Eq. 2, $\phi_{\mathrm{obs}}^{\mathrm{atm}}(\lambda)$ and $\phi_{\mathrm{b}, \mathrm{obs}}^{\mathrm{inst}}(\lambda)$ are the atmospheric and instrumental ${ }^{2}$ components of $\phi_{\mathrm{b} \text {,tot }}(\lambda)$ at the location of the source on the focal plane from Eq. 2 such that $\phi_{\mathrm{obs}}^{\mathrm{atm}}(\lambda) \phi_{\mathrm{b} \text {,obs }}^{\mathrm{inst}}(\lambda)=\phi_{\mathrm{b} \text {, tot }}(\lambda)$, and $\phi_{\mathrm{ref}}^{\mathrm{atm}}(\lambda)$ and $\phi_{\mathrm{b}, \text { ref }}^{\text {ins }}(\lambda)$ are the reference atmospheric and DECam transmission functions within a given band, $b$, respectively. The reference transmission functions are chosen during the FGCM process to represent the most probable conditions over the course of the survey (see Fig. 4 in B18).

Due to this choice of reference transmission, the average chromatic correction for a single object over an infinite number of observations should trend to zero. However, SNe Ia are time varying and the shape of the light curve is important for standardization. Therefore, trends in atmospheric parameters that depend on time (e.g seasonal variations, El Niño, and degradation of the primary mirror) could produce effects that will not average to zero. The light curve sampling requirements result in non-uniform sampling of events over the course of the survey and therefore seasonal variations in atmospheric properties could potentially result in chromatic corrections whose effect on SN Ia distance does not average to zero.

The correction in Eq. 3 is defined so that $\delta_{b}^{m}$ is equal to zero for observations of the reference source with the reference transmission function. The atmospheric transmission functions are informed by our PWV measurements and the DECam transmission functions are measured by the DECal scans with additional input on the focal plane variation from star flats. The $\delta_{b}^{m}$ correction is added to the zeropoint based on the SED of the source. While applying these corrections reduces the overall calibration uncertainty, we have not separated out the uncertainty contribution from the atmospheric transmission function, focal plane transmission functions, or the SNIa SED flux uncertainty. The part of the uncertainty due to the transmission function alone is included in the calibration uncertainty in B18.

These chromatic corrections are an improvement over the previous linear atmospheric correction ${ }^{3}$ in two major ways. First, they account for variation in the atmospheric conditions over the course of each night of observing whereas the linear correction coefficients were fit nightly. Second, the chromatic corrections incorporate SED information allowing for the correction of non-blackbody spectra and objects whose spectra have strong features

Using a small data sample, L16 shows that the effect of these chromatic corrections on SNe Ia can be as large as $10 \mathrm{mmag}(1 \%)$

2 telescope, instrument, filter, and CCD

3 http://classic.sdss.org/dr7/algorithms/jeg_photometric_eq_dr1.html in $z$-band and several mmag in $r$ and $i$ bands for high redshifts and large atmospheric water vapor. This study illustrates that for SEDs that differ significantly from the reference, the chromatic corrections can be comparable or larger than to the non-uniformity of the calibration $(\approx 4.5 \mathrm{mmag})$.

The middle row of panels in Fig. 7 from L16 shows that the variation in the corrections described in Eq. 3 matches the observed variation in stellar magnitude vs. stellar color to mmag precision. The typical color range observed in SNe Ia is $0.5<$ $g-i<3.5$, which includes the entire range of that figure. This test demonstrates that chromatic corrections improve the calibration for sources whose SED differs from the reference SED.

\subsection{Data sample}

The Dark Energy Survey includes a $5000 \mathrm{deg}^{2}$ ("wide") survey (Diehl et al. 2018) and a $27 \mathrm{deg}^{2}$, time domain, supernova survey (Bernstein et al. 2012; Kessler et al. 2015), which are run concurrently between August and February beginning in 2013 and ending in 2018. The wide survey alone will continue operations into 2019. The wide survey is conducted in 5 bands ( $\operatorname{riz} Y$ ) of which the 4 bluest bands ( griz) are used in the supernova survey. Survey observations are conducted on the Victor Blanco $4 \mathrm{~m}$ telescope using the Dark Energy Camera (DECam) at the CTIO in Chile.

The supernova fields are observed when the predicted point spread function (PSF) is above 1.1" or when a field hits a "deadman" trigger meaning that it has not been observed for 7 days. The atmospheric conditions of the supernova survey are illustrated in Fig. 2, whose three panels shows the distribution of PWV, atmospheric optical depth due to aerosols $(\tau)$, and PSF respectively. While PWV and $\tau$ are comparable to the median DES wide area conditions, the median PSF is about a tenth of an arcsecond above the median PSF of the wide area survey.

Atmospheric parameters PWV and $\tau$ were computed by B18 for exposures satisfying quality requirements for the wide-area, and thus $10 \%$ of supernova survey observations do not have the atmospheric parts of the correction. However, all exposures are corrected for instrumental transmission variation. Atmospheric information for all exposures will be included in a future paper that will cover the calibration of the full five seasons of DES.

The DES SNe are discovered in the "real-time" difference imaging pipeline (DIFFIMG, Kessler et al. 2015), where deep coadded template images are subtracted from each supernova survey image. In this paper we use 214 spectroscopically confirmed $\mathrm{SNe}$ Ia discovered from the first three years of DES-SN. The spectroscopic selection of the sample is described in DâĂŹ'Andrea et al (2018, in prep.). The $2 \%$ calibration uncertainty for DIFFIMG photometry is sufficient for SN discovery and monitoring, but is not sufficient for the cosmology analysis. Therefore, in this analysis we use a version of "scene modeling" photometry (SMP, Brout et al. 2018a, in prep.) developed by DES for use in the offline analysis with the goal of achieving sub-percent precision. This method was originally developed by SNLS (Astier et al. 2006) and later used by SDSS (Holtzman et al. 2008).

The calibration of SMP photometry uses an arbitrary reference transmission function, which we choose to be the focal plane averaged reference transmission function. Next, the chromatic corrections are applied to all observations so that their photometry is on a single system defined by the reference transmission function. While this addresses the chromatic effects, it does not address other filter transmission-related systematics such as uncertainties in the 
central wavelength of the transmission functions. The effect of this systematic is discussed in Brout et al. 2018b $\S 4.1$.

To improve the cosmological parameter determination, the spectroscopically confirmed subset of the DES-SN sample is combined with 126 low redshift (low- $z$ ) supernovae from surveys including CFA3, CFA4, and CSP. This sample is taken from the Pantheon analysis (Scolnic et al. 2017) with additional cuts described in DES Collaboration 2018. We do not apply chromatic corrections to the low- $z$ sample because we do not have the information necessary to make these corrections. Instead, we use the original survey calibration.Since the low redshift events do not include $z$-band data, they are not affected by the strongest component of the atmospheric effect, the water vapor absorption band near $900 \mathrm{~nm}$. The focal plane portion of the chromatic effects are partly removed by the Supercal analysis (Scolnic et al. 2015) which puts the lowz sample onto the PS1 system. The combination of the DES-SN sample and the low- $z$ sample is referred to as "DES-SN3YR."

In order to study the effect of chromatic corrections with large statistics in all areas of parameter space (e.g. SN parameters like redshift, color, and stretch as well as observing conditions like $\tau$ and PWV), we utilise a DES-SN3YR-like sample produced by the simulation code in the SuperNova ANAlysis (SNANA ${ }^{4}$, Kessler et al. 2009, Kessler et al. 2018) software package. We use this simulation to generate a large number of SEDs, approximately 120x the size of the DES-SN3YR sample, for which we can assess the impact of chromatic corrections.

These simulated supernovae are generated using the color and stretch distributions of Scolnic \& Kessler (2016), the volumetric rate from Perrett et al. (2012), the spectroscopic selection function from D'Andrea et al. 2018, host galaxy library from Gupta et al. (2016), and the intrinsic scatter model from Guy et al. (2010); Kessler et al. (2013). This simulation uses randomly chosen sky coordinates over the supernova fields, selects a random CCD from the focal plane, and uses DES observation dates. The date and focal plane location are used to determine chromatic corrections (Eq. 3) in the same manner as for the data.

Fig. 3 shows the redshift and maximum signal to noise ratio (SNR) distributions for the DES-SN sample. The simulations agree well with the data for the DES-SN sample. A similar plot for the low- $z$ sample is shown in Fig. 7. of Kessler et al. 2018

\subsection{Light-curve and cosmology fitting}

The SNANA software package provides light-curve fitting code using the Spectral Adaptive Lightcurve Template 2 (SALT2) model first developed by Guy et al. (2007). We use the most recently trained SALT2 model that was developed for the Joint Light-curve Analysis (JLA, Betoule et al. 2014). However, to see the effect of the chromatic corrections on $z$-band in the lowest redshift DES supernovae, we use the Near Infrared (NIR) extension of this model from Hounsell et al. (2017). The light-curve fitting code determines the stretch $\left(x_{1}\right)$, color $(c)$, amplitude $\left(x_{0}\right)$, and time of peak brightness $\left(t_{0}\right)$ for each supernova light-curve, both for the DES-SN3YR data sample and the simulated sample described in $\$ 2.2$. Distance moduli are calculated by the Tripp estimator (Tripp 1998):

$\mu=M_{0}+m_{B}+\alpha x_{1}-\beta c$,

4 snana.uchicago.edu for manual and other information where $m_{B}=-2.5 \log _{10}\left(x_{0}\right), \alpha$ is the stretch-magnitude standardization parameter, and $\beta$ is the color-magnitude standardization parameter.

In the first step of the analysis, the light curves are fit without chromatic corrections to determine the SED at each epoch using the SALT2 spectral model. Next, the light curves are fit with corrections (Eq. 3) applied.In both cases the synthetic photometry used in the light curve fit is based on the single reference transmission function, and not an epoch dependent transmission function.

After light curve fitting, the standardization parameters $\alpha$ and $\beta$ and a Hubble diagram which is corrected for biases due to selection effects and light curve fitting are determined simultaneously from a global fit to the set of DES-SN3YR light curve parameters $\left(c, x_{1}, m_{B}\right)$. This global fit is performed with the BEAMS with Bias Correction (BBC, Kessler \& Scolnic 2017) formalism with 20 logarithmically spaced redshift bins from 0.01 to 0.85 .

The binned distances and uncertainties are passed to wFit, a fast $\chi^{2}$ minimization program using MINUIT (James \& Roos 1975), which outputs marginalised cosmology parameters $w$ and $\Omega_{m}$ based on a $w$ CDM model, a flat universe with varying dark energy equation of state parameter, $w$, and cold dark matter. These parameters are obtained with priors from BAO (Eisenstein et al. 2005) and CMB (Komatsu et al. 2009). The cosmological parameters are blinded so that we only examine differences due to the chromatic corrections. These simplifications are used because they are significantly faster and sufficiently accurate for differential studies. However, we do not use these simplifications in the nominal DESSN3YR cosmology analysis (DES Collaboration 2018).

For each SN, we calculate the change in the BBC distance modulus $\mu$ and the three parameters $x_{1}, c$, and $m_{B}$ due to the chromatic corrections. The light-curve fit parameters $x_{1}$ and $c$ are multiplied by the nuisance parameters $\alpha$ and $\beta$ to give them the same units (mag) as $\mu$ and $m_{B} . \alpha$ and $\beta$ are fit separately with BBC before and after corrections are applied; however, they do not significantly change due to the corrections. Therefore, we adopt a single value for alpha and beta when calculating the differences. These differences are defined below:

$\Delta \mu=\mu_{\mathrm{noCorr}}-\mu_{\mathrm{corr}}$

$\Delta \alpha x_{1}=\alpha x_{1, \mathrm{noCorr}}-\alpha x_{1, \mathrm{corr}}$

$\Delta \beta c=\beta c_{\mathrm{noCorr}}-\beta c_{\mathrm{corr}}$

$\Delta m_{B}=m_{B, \text { noCorr }}-m_{B, \text { corr }}$.

We characterise the $\Delta$ parameter dependence on redshift using a linear fit ( $\Delta$ vs. redshift) to the unbinned SN sample in order to obtain a simple one parameter quantification of the effect of the corrections. These values and their slopes can be seen in Figs. 6, 7,8 , and 12 . Since the fitting uncertainty on the slopes of the bestfit lines does not account for correlations (e.g. between $x_{1 \text {,noCorr }}$ and $x_{1 \text {,corr }}$ ), the uncertainty is determined empirically. We generate 50 data-sized simulations of the DES-SN3YR sample. Then, after running those samples through the same analysis as the data, we collect the fitted values of the $\Delta$ parameter slopes vs. redshift and $\Delta$ cosmological parameters. We use the standard deviation of the slopes and cosmological parameters among the 50 simulations 

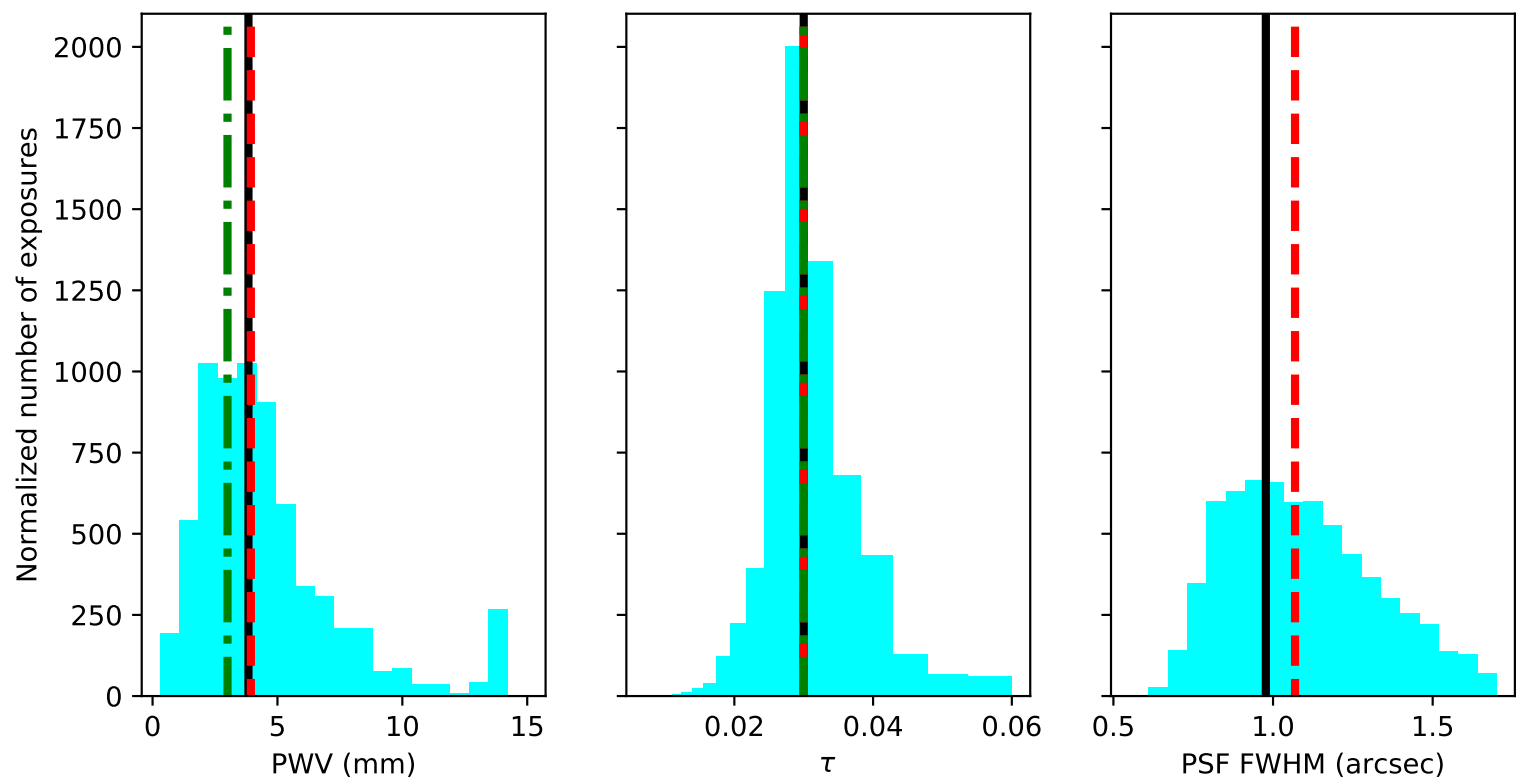

Figure 2. The distribution of atmospheric precipitable water vapor (PWV), optical depth due to aerosols $(\tau)$, and PSF FWHM for DES observations of the supernova fields during the first three seasons. The black solid and red dashed lines represent the median conditions over the first three seasons of the survey for DES and DES-SN respectively, and the green dotted and dashed line represents the reference atmosphere (there is no standard PSF size). All four bands (griz) are included.
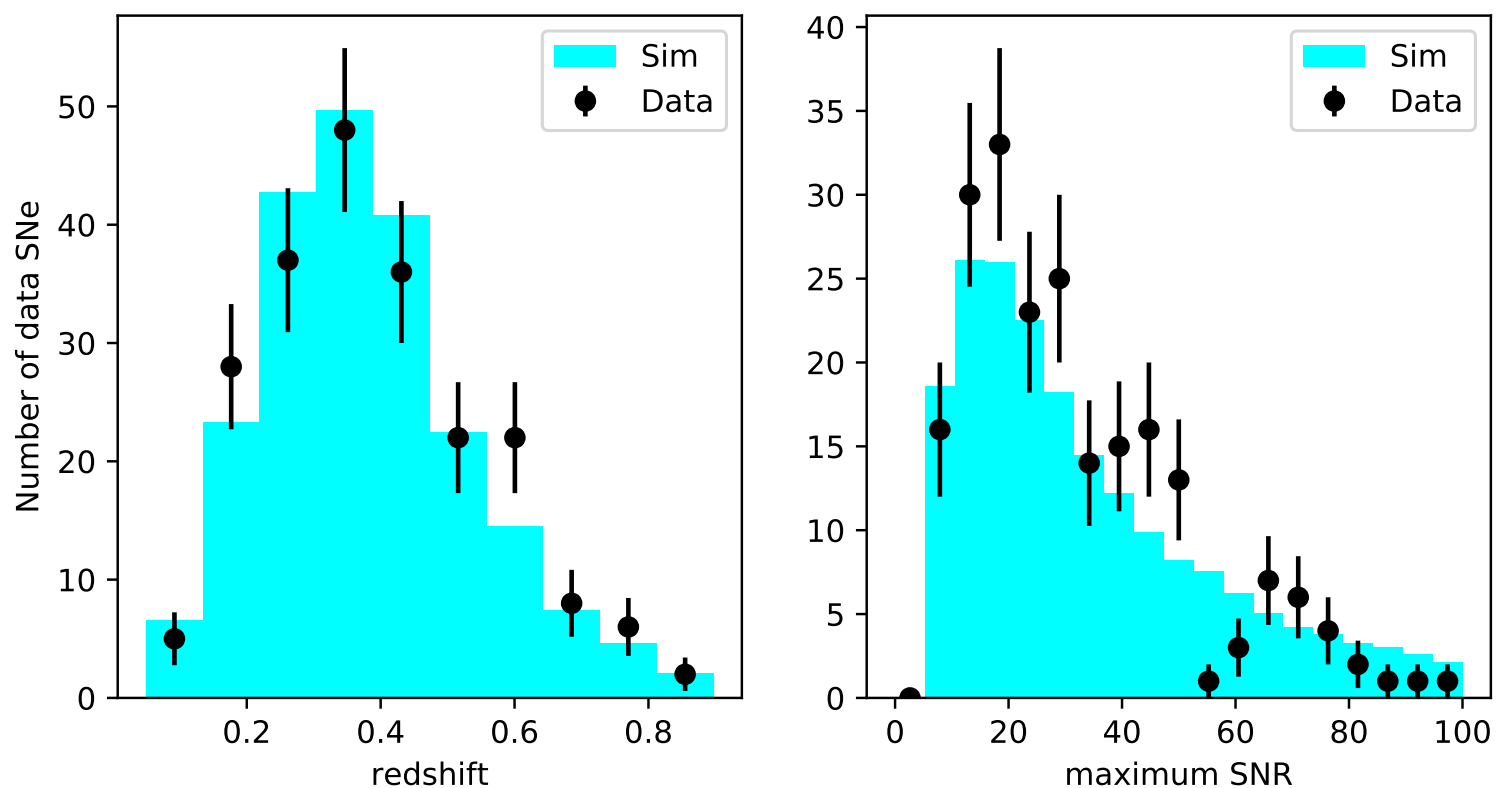

Figure 3. The distribution of redshift (left) and maximum signal to noise (right) for SNe Ia in the DES-SN data sample (black circles with error bars) and simulation (light blue bars). 
to estimate the uncertainty. We believe this is valid since the distribution of these 50 slopes is consistent with a normal distribution. We also use those uncertainty estimates for the larger simulated sample. However, for that larger sample we scale down the uncertainty by the square root of the ratio of the size of the larger sample to the size of the DES-SN3YR sample. Applying corrections based on these linear relationships is not a substitute for applying the full integrated correction to each supernova epoch. However, calculating the slopes is useful to check whether the simulated SNe change similarly to the data events as well as to check whether the redshift trend (or lack thereof) in parameter changes indicates that there should or should not be a cosmological parameter bias.

We define changes in the wFit output cosmological parameters $w$ and $\Omega_{m}$ :

$\Delta w=w_{\text {noCorr }}-w_{\text {corr }}$

$\Delta \Omega_{m}=\Omega_{\mathrm{m}, \text { noCorr }}-\Omega_{\mathrm{m}, \text { corr }}$.

Following the method for determining the uncertainties on the slopes above, the uncertainties on $\Delta w$ and $\Delta \Omega_{m}$ are the standard deviation in these quantities from 50 DES-SN3YR sized simulations. These uncertainties are also scaled by the square root of the sample size.

\section{RESULTS}

We begin this section with the impact of the corrections on the single-epoch photometry and show the dependence on SN color, redshift, and atmospheric PWV. Next we show a comparison of the SALT2 nuisance parameters $\alpha$ and $\beta$ between this analysis and the Pantheon analysis, as well as the change in those parameters due to the chromatic corrections. Finally, we present the changes in lightcurve fit parameters, distance moduli, and cosmological parameters due to these corrections.

\subsection{Impact on single-epoch photometry}

We apply the corrections described in $\$ 2.1$ to the DES-SN3YR sample and examine the effects on single-epoch photometry. Since the $z$ band includes water absorption lines, we present those results here. The $g, r$, and $i$ bands show a median chromatic correction consistent with zero at all values of redshift, PWV, and observed $r-i$ (for $g$ and $r$ band) or $i-z$ (for $i$ band) color. The standard deviation of all chromatic corrections in $g, r$, and $i$ bands respectively are $11.1,3.3$, and 4.4 mmag.

The upper left panel of Figure 4 shows the average ( $z$-band) chromatic correction as a function of PWV and $i-z$ color when applied to the DES-SN3YR sample. PWV and $i-z$ are divided into 10 evenly spaced bins over the range of observed parameter space. Those panels are further subdivided into panels based on the $\mathrm{SN}$ redshift. These plots include all SN epochs regardless of phase relative to peak brightness in the model $\mathrm{B}$ band.

There is a trend of about $1 \mathrm{mmag}$ per $\mathrm{mm}$ of PWV at low redshifts and that trend reverses to -1 mmag per mm of PWV at highest redshifts. To see the $\delta_{z}^{m}$ effect with higher statistics, the upper right panel of Figure 4 shows a prediction using a simulation of 120 DES-SN3YR samples. This simulation confirms the trend observed in the data. There is no statistically significant trend with light-curve fit color in data or simulation. The data sample appears
Table 1. BBC nuisance parameters for DES-SN3YR and Pantheon samples.

\begin{tabular}{cccc}
\hline Dataset & $\alpha$ & $\beta$ & $\sigma_{\text {int }}$ \\
\hline DES Uncorrected $^{0}$ & $0.144 \pm 0.008$ & $3.12 \pm 0.104$ & 0.097 \\
\hline DES Corrected $^{5}$ & $0.145 \pm 0.008$ & $3.11 \pm 0.10$ & 0.097 \\
\hline Pantheon & $0.156 \pm 0.006$ & $3.02 \pm 0.06$ & 0.09 \\
\hline
\end{tabular}

to have very low scatter in some PWV/color+redshift bins because it only has one or two events that fall in that bin. The simulated scatter is more representative of the true scatter in the chromatic corrections.

In order to further illustrate the effect of the chromatic corrections due to atmospheric and CCD variations, in Fig. 5 we present the distribution of corrections for two selected SN SEDs integrated for each atmospheric transmission function observed during DES. These SN SEDs are from Figs. $1 \mathrm{~b}$ and $1 \mathrm{c}$, with redshifts 0.36 and 0.85 , respectively. We present two panels for each of the two sample SEDs: the first set of panels takes its instrumental transmission function from 6 interior CCDs and the other takes its instrumental transmission function from the 6 outer CCDs. The median of the chromatic correction distribution is significantly different when considering the inner CCDs vs. the outer CCDs and the shape of the distribution is much wider for the lower redshift SN than the higher redshift SN.

The width of the low-redshift chromatic correction distribution is driven primarily by PWV variations between 0.5 and $15 \mathrm{~mm}$. Within the z-band wavelength range, the AB spectrum is nearly flat, while the SN spectra are significantly more tilted, and thus PWV variations, which affect the region near $\lambda \sim 9500 \AA$, have a larger effect on the $\mathrm{AB}$ spectrum. The distribution for high redshift (lower panels) is much narrower due to the fortuitous bump in the exact location of the PWV feature. We have checked that without this bump, the width of the chromatic correction distribution is much larger and also reproduces the secondary peak that we observe in the low redshift distribution.

\subsection{Result for BBC fitted SALT2 nuisance parameters}

Table 1 shows the nuisance parameters $\alpha$ and $\beta$ from the BBC fits of the DES-SN3YR sample, as well as those from the Pantheon Sample. We compare these parameters to check our fitting method without unblinding the cosmological parameter fit. $\alpha$ and $\beta$ are statistically consistent between DES-SN3YR and Pantheon, and the chromatic corrections result in negligible shifts $(<1 \%)$. The table also includes the intrinsic scatter of supernova brightness $\left(\sigma_{\text {int }}\right)$ which is calculated as the amount of additional error that needs to be added during the fit to get the reduced $\chi^{2}$ to be equal to one. This value is also comparable to the scatter in Pantheon (Scolnic et al. 2017).

\subsection{Effect of chromatic corrections on light-curve fit parameters, distances, and cosmology}

Here we propagate both the DES-SN3YR and simulated samples through the analysis and show the effects of the chromatic corrections as a function of redshift on the fit parameters.

Figure 6 shows the redshift dependence of the effect of the 

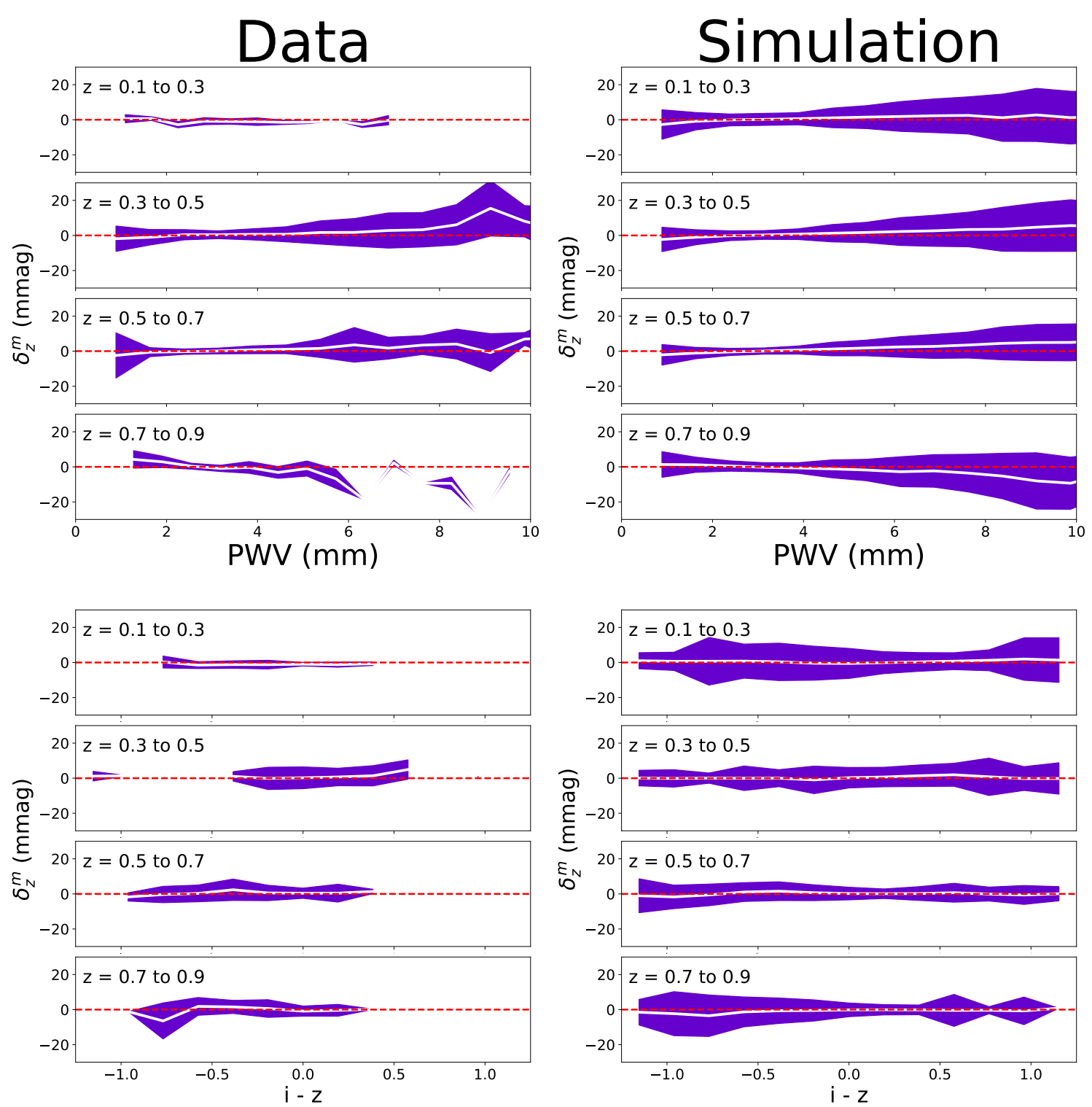

Figure 4. For DES $z$ band, $\delta_{z}^{m}$ dependence on PWV (top) and $i-z$ color (bottom). Each set of 4 panels shows a different redshift range for data (left) and simulations (right). The white solid lines connect the median chromatic correction in each PWV/color bin, the red dashed line is zero, and the colored band represents the standard deviations within each bin.

chromatic corrections on the measured distance modulus $(\Delta \mu)$, as well as on the light-curve fit parameters $\left(\Delta x_{1}, \Delta c\right.$, and $\left.\Delta m_{B}\right)$ for the data. The slopes of the shift vs. redshift given on the top of each panel show that each slope is consistent with zero.

Figure 7 shows the same quantities as in Fig. 6, but for the simulated DES-SN3YR sample, which has slope uncertainties that are almost an order of magnitude smaller than those of the data. For the simulated $\mathrm{SNe}, \Delta m_{B}$ shows a nonzero slope with 3- $\sigma$ significance $(0.6 \pm 0.2 \mathrm{mmag})$. This effect does not propagate to any significant redshift trend in distance modulus vs. redshift. The re- maining light-curve fit parameters have slopes vs. redshift that are consistent with zero at a 1- $\sigma$ level. All of the slopes for the simulated sample parameters are consistent with the slopes of the data sample parameters as shown in the top two rows of each panel of Fig. 8. While the mean of the chromatic corrections is small, the scatters in these plots exhibit the range of the chromatic corrections on the individually measured distance moduli and fitted light-curve parameters.

To examine the relative effects of the atmospheric and instrumental corrections, we made the corrections for the simulated sam- 

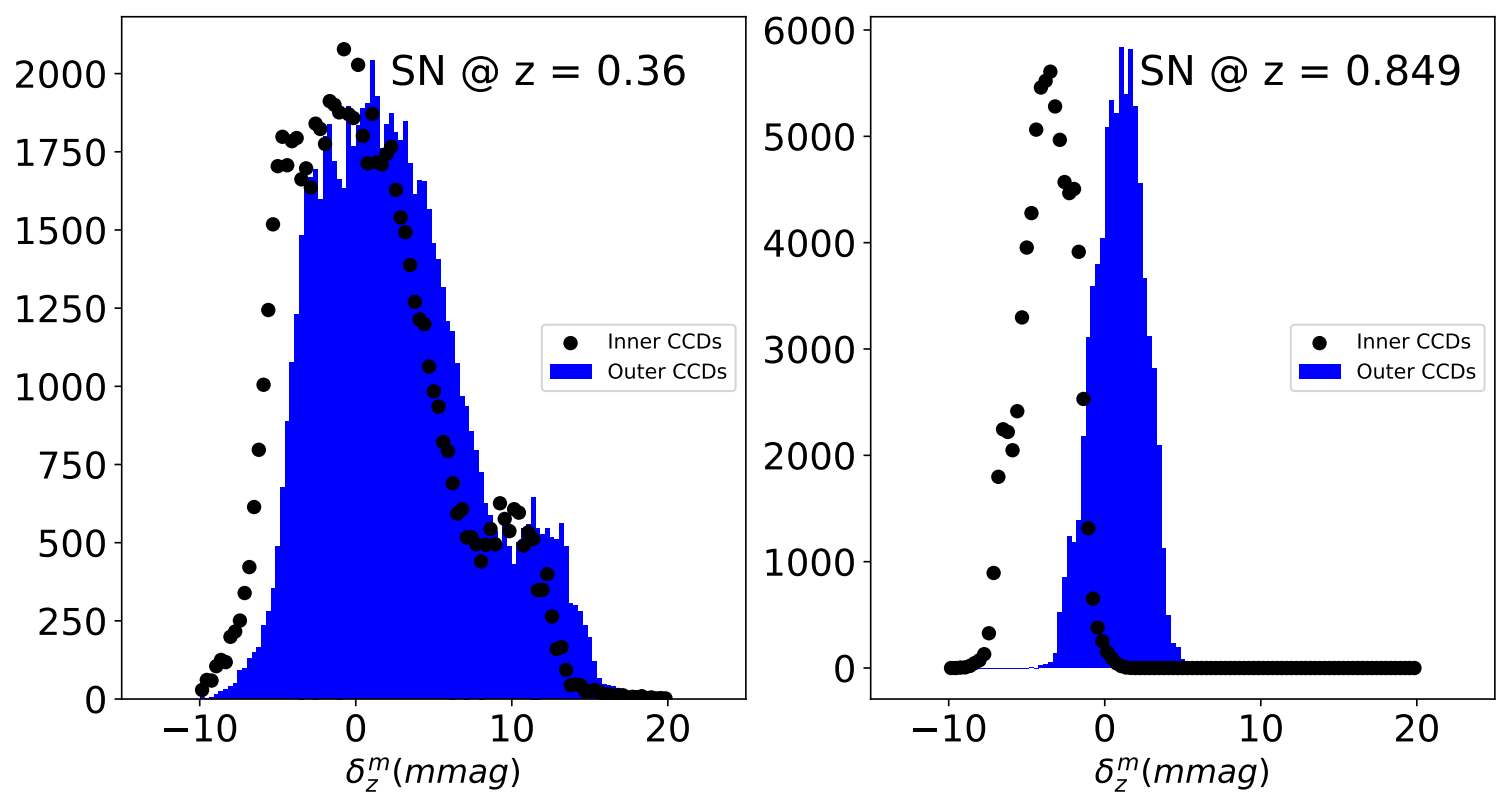

Figure 5. For DES $z$ band, $\delta_{z}^{m}$ distribution for the SN spectrum in Fig. $1 \mathrm{~b}$ (left) and Fig. $1 \mathrm{c}$ (right). Each set of panels shows a different set of CCDs (inner CCDs as black dots and outer CCDs as blue bands).

ple using the standard atmosphere, zeroing out the atmospheric correction, and then we made a second set of corrections that are the differences between the full (atmospheric + instrumental) corrections and the instrumental only corrections. The effect of these corrections on distance modulus are shown in Fig. 9 and Fig. 10 below.

These figures (Fig. 9 and 10) show that the trend in distance correction vs. redshift is mostly due to the atmospheric effects, but the oscillatory features are mostly due to the instrumental effects. We have examined the trend of $\Delta \mu \mathrm{vs}$. redshift for individual CCDs, and we find that the oscillations are present in each CCD and are not an artifact of stacking all of the CCDs.

For the data, the shifts in the cosmological parameters $\Omega_{m}$ and $w$ due to the chromatic corrections $\left(\Delta \Omega_{m}\right.$ and $\Delta w$ as in Equations 9 and 10) are $\Delta w=-0.002$ and $\Delta \Omega_{m}=0.000$. These changes are consistent with our simulated results where the mean change in $w$ over our 50 simulated DES-SN3YR-sized simulations is 0.007 with an standard error in the mean of 0.008. Similarly, the mean $\Omega_{m}$ change is 0.001 with a standard error in the mean of 0.001 . The simulation results are consistent with zero as expected. They are also consistent with the data based on our limited sample size. The results are visualised in the top two rows of each panel of Fig. 11.

For an SN Ia-only analysis, we find $\Delta \Omega_{m}$ and $\Delta w$ are 0.005 and -0.0294 . However, since the shift occurs along the direction of the SN Ia-only contour degeneracy, the effect on the combined $\mathrm{SN}$ Ia, CMB, and BAO results are negligible for the DES data set. Furthermore the shift is still negligible in an SN Ia-only analysis relative to the parameter uncertainties, 0.07 and 0.35 respectively for $\Omega_{\mathrm{m}}$ and $w$.

\subsection{Results on individual CCDs}

There is no significant trend in $\Delta \mu$ vs redshift as shown in Figs. 6 and 7. However, this is not the case for individual CCDs in the simulated sample. The data sample is too small to get meaningful results for individual CCDs, so we use a simulated sample. Fig. 12 shows $\Delta \mu$ vs redshift for 4 different CCDs at different distances from the centre of the focal plane. CCD 35 is near the center, CCD 52 is halfway between the centre and the edge, and CCDs 1 and 62 are at the far edge of the focal plane on opposite sides. These 4 CCDs were chosen to sample the radial transmission function variation as shown in L16. These simulations show that some CCDs have a strong trend in $\Delta \mu$ vs. redshift.

The results of fitting these redshift trends for the data, simulation, and subsets of the simulation for each of the chosen CCDs are summarised in the bottom four rows of each panel of Fig. 8. The scatter among the individual CCD samples is large and in many cases the CCDs are both inconsistent with each other and with zero. The individual corrections show a strong oscillatory behavior with redshift that comes from features of the SED moving into and out of the bandpasses with redshift.

\subsection{Cross checks}

Since our redshift cutoff of 0.85 is a function of the DES spectroscopic selection function (D'âĂŹAndrea et al 2018 in prep.) and is not related to the chromatic corrections or the supernovae themselves, we have also tested the effect of changing this cutoff to lower redshift. There is no significant change in $\Delta w$ and $\Delta \Omega_{m}$ with decreasing redshift cutoff.

To obtain the spectra used in the chromatic corrections, we use the spectral templates from the SALT2 model. This SED model is constructed from spline basis functions and thus may alter some of the SN spectral features. To check if our chromatic corrections are sensitive to the SALT2 SED representation, we have performed a cross-check based on the spectral time series created by Hsiao et al. (2007). Mosher et al. (2014) constructed a model from the Hsiao spectral time series using the SALT2 stretch and color law relations while preserving the spectral features. Using this model results in 

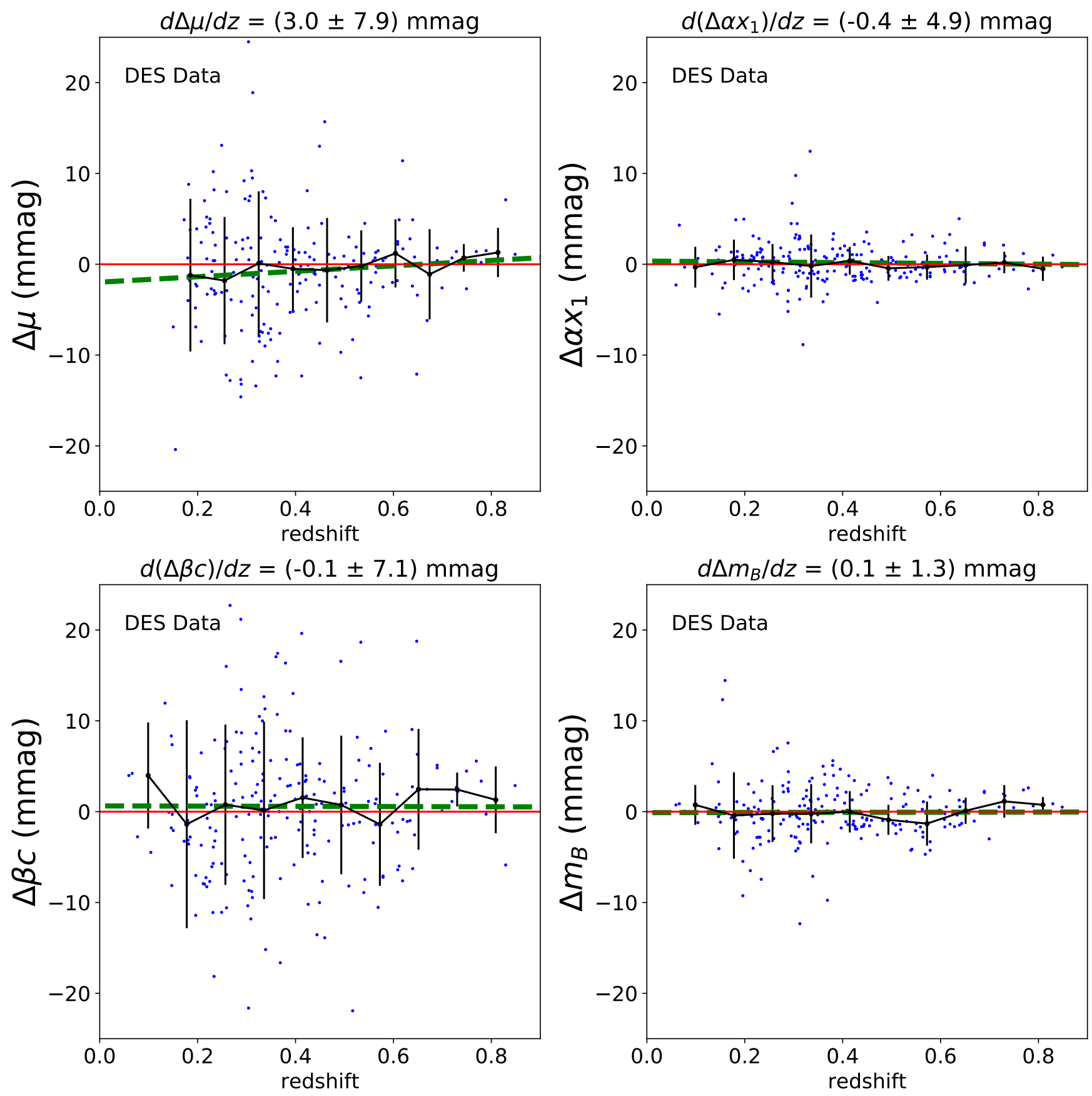

Figure 6. The redshift dependence of $\Delta \mu, \Delta \alpha x_{1}, \Delta \beta c$, and $\Delta m_{B}$ (Eqs. 5, 6, 7, and 8 respectively) for the DES-SN3YR SN Ia sample. Each dot is an individual SN from the DES-SN3YR sample. The black line connects the median $\Delta$ for the SNe in each redshift bin, its error bars represent the standard deviation of the chromatic correction in each bin, the red solid line is zero, and the green dotted line is the best-fit line whose slope and uncertainty are given above each panel.

corrections consistent with those based on the SALT2 model spectra: the $\Delta \mu$ (Eq.5) agree to within $0.25 \mathrm{mmag}$ for $-0.3<c<+0.3$.

It is known that the weather in the first half of the DES seasons (September-November) is usually worse than that in the second half of the seasons (December-February) at CTIO. Therefore we split our simulated sample into halves of seasons and performed the same analysis that was performed on the entire sample above. We find that the average change in $w$ between the first and second half of seasons is $0.006 \pm 0.016$, an insignificant effect. In addition, we split our data into years (Year 1: 2013-14, Year 2: 2014-15, Year $3: 2015-16)$ in order to assess any potential changes in cosmological parameters between years, specifically Year 3 which was heavily affected by El Niño. We did not find any measurable change in either $w$ or $\Omega_{m}$. 

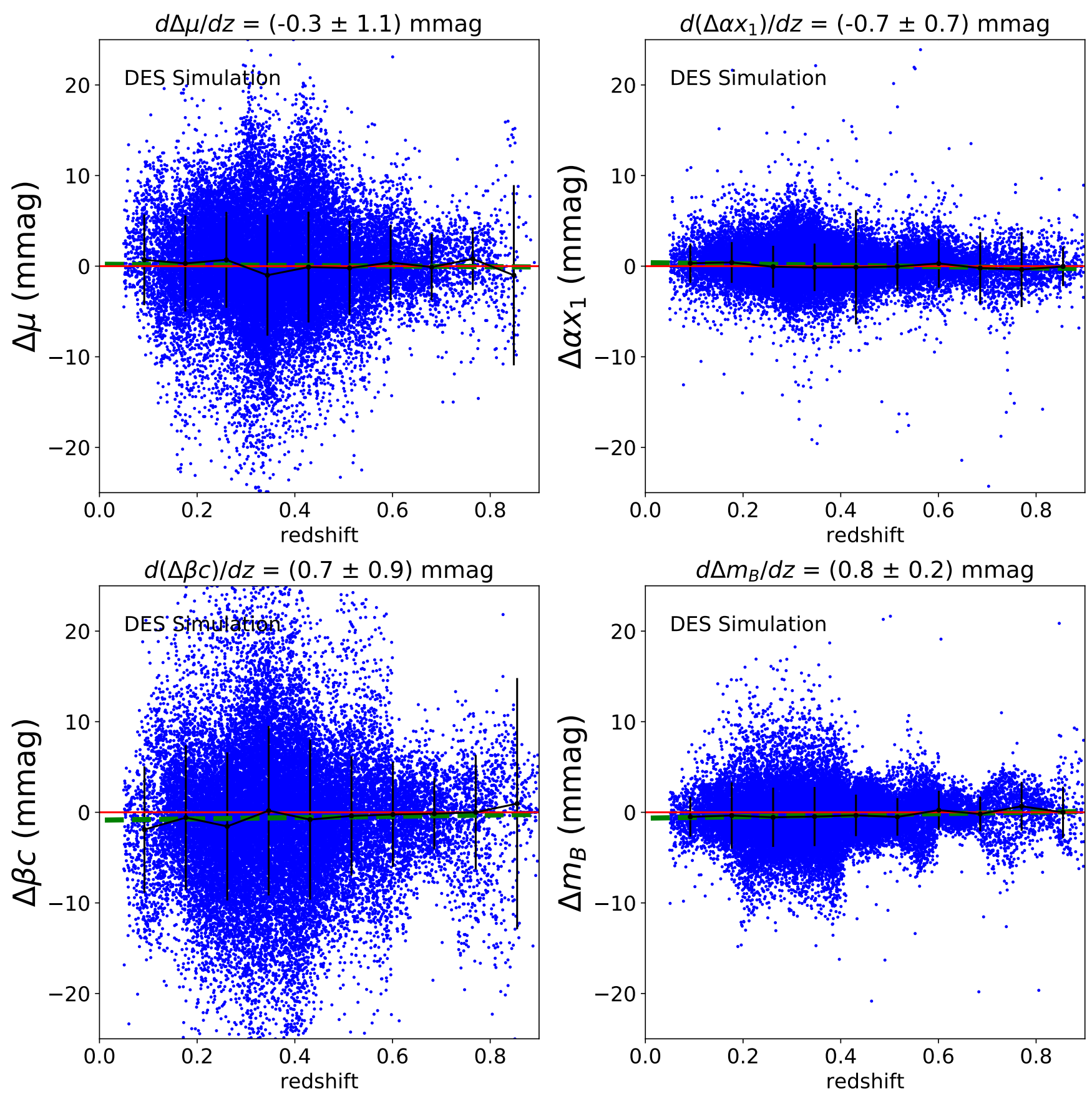

Figure 7. Same as Fig. 6, but for the simulated DES-SN3YR-like sample.

\section{CONCLUSION}

In this paper, we presented the first application of the chromatic corrections described in B18 to type Ia supernova cosmology. We applied the corrections to the DES-SN3YR supernova sample as defined in the DES Collaboration 2018 analysis. The effect of the chromatic corrections on distance modulus is not significant in either data or simulation. The $1 \sigma$ limit on the median size of the chromatic correction on the single epoch photometry is a less than 2 mmag change in correction over the redshift range from $z=0$ to $z=1$. This limit is valid for the DES-SN3YR sample and is not necessarily valid for other samples, although this has not been tested.

The application of chromatic corrections, while necessary to achieve the precision photometry in $\mathrm{B} 18$, results in a change in $w$ of $-0.002 \pm 0.008$ and a change in $\Omega_{m}$ of $0.000 \pm 0.002$, for the combined SN Ia, BAO, and CMB analysis, which is not statistically significant. Examining the effect of the corrections on single CCDs both in $\Delta \mu$ trends vs. redshift and cosmological parameters shows that this effect would become significant on a targeted survey where the observations are placed on a single CCD or subset of CCDs. This is assuming that such a survey would use the FGCM 

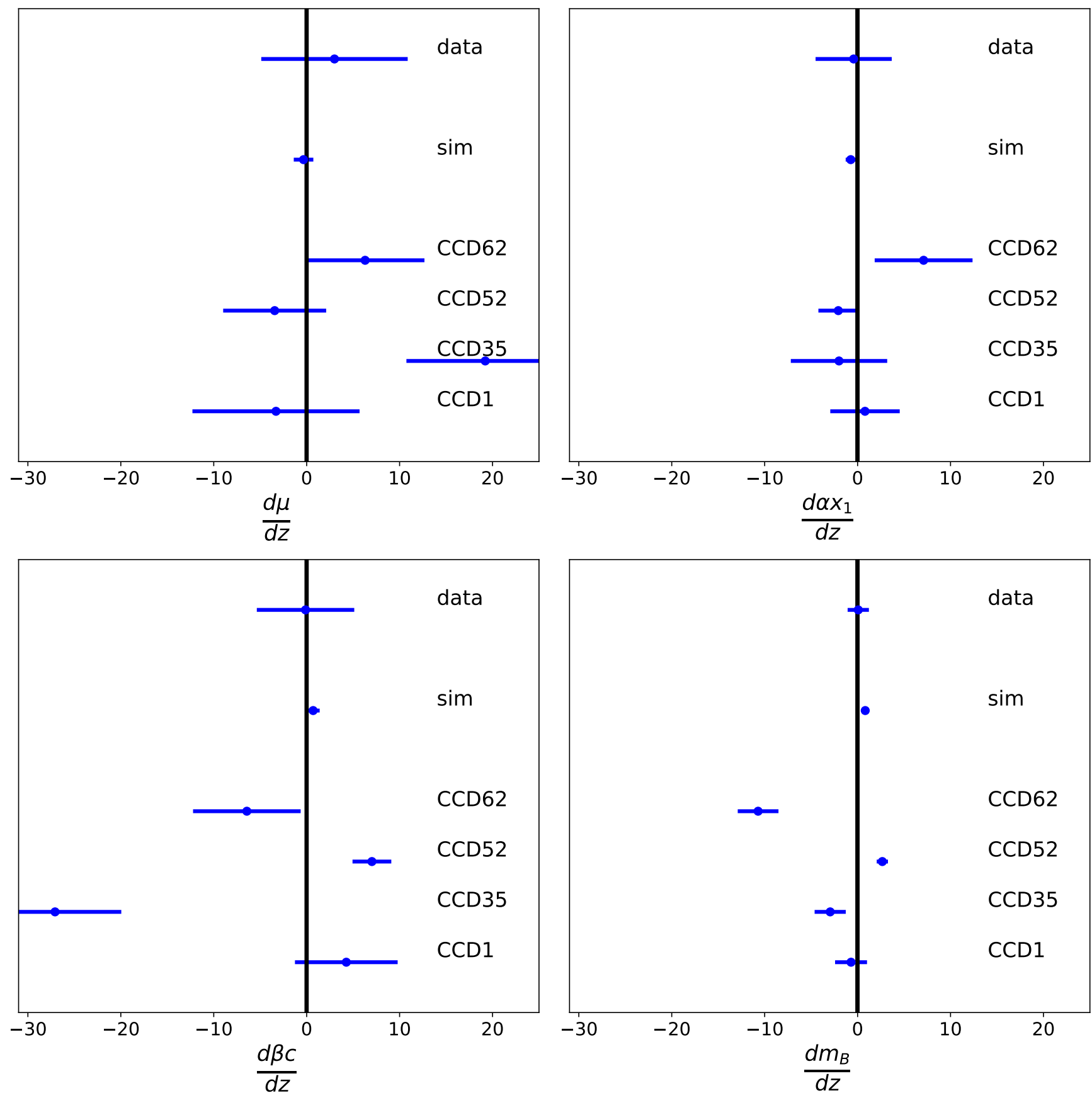

Figure 8. The slope of the $\Delta \mu, \Delta \alpha x_{1}$ (upper right), $\Delta \beta c$ (lower left), and $\Delta m_{B}$ (lower right) (Equations 5, 6, 7, and 8) vs. redshift for the data sample, simulated sample, and subsets of the simulated sample for 4 individual CCDs.

calibration of DES and would not recalibrate itself to a reference spectrum based only on the CCDs it used. Despite this analysis showing that for DES, the atmospheric-only chromatic corrections average out to have no overall effect, this is not a general proof that this is true for all surveys. For future surveys such as LSST, we cannot make predictions for the impact of chromatic corrections. However, we note that LSST will observe year-round and therefore seasonal variations may be more significant compared with those from the five month observing seasons of DES.

\section{ACKNOWLEDGEMENTS}

James Lasker is grateful for support from the ARCS (Acheivement Rewards for College Scholars) foundation and its donors. James Lasker, Rick Kessler, Dan Scolnic, and Josh Frieman are grateful for the support of the University of Chicago Research Computing Center for assistance with the calculations carried out in this work and to the Kavli Institute for Cosmological Physics.

Dan Scolnic is supported by NASA through Hubble Fellowship grant HST-HF2-51383.001 awarded by the Space Telescope 


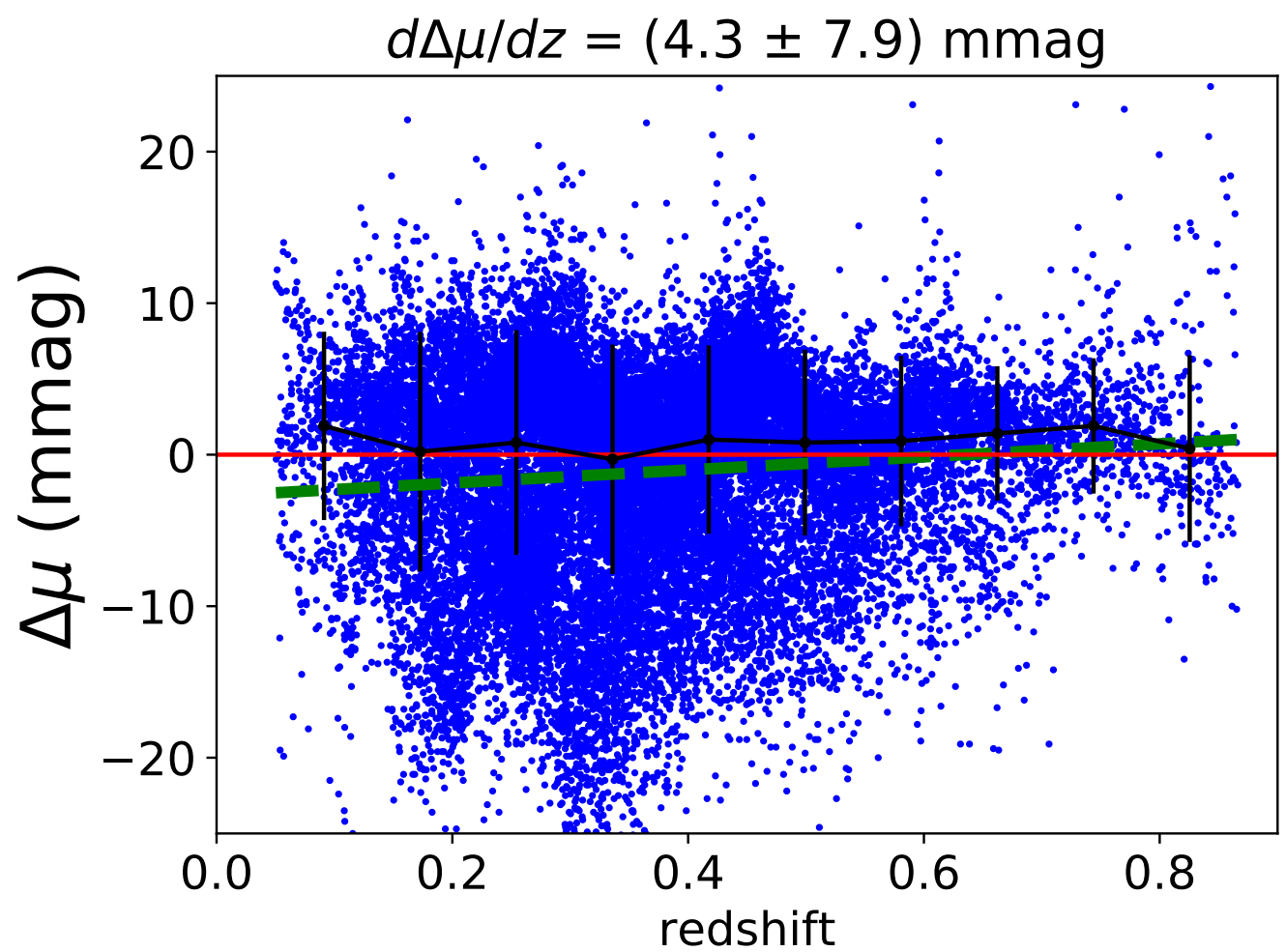

Figure 9. Same as Fig. 7 upper left panel, but for atmospheric corrections only, and only for the four chosen CCDs $(1,35,52,62)$.

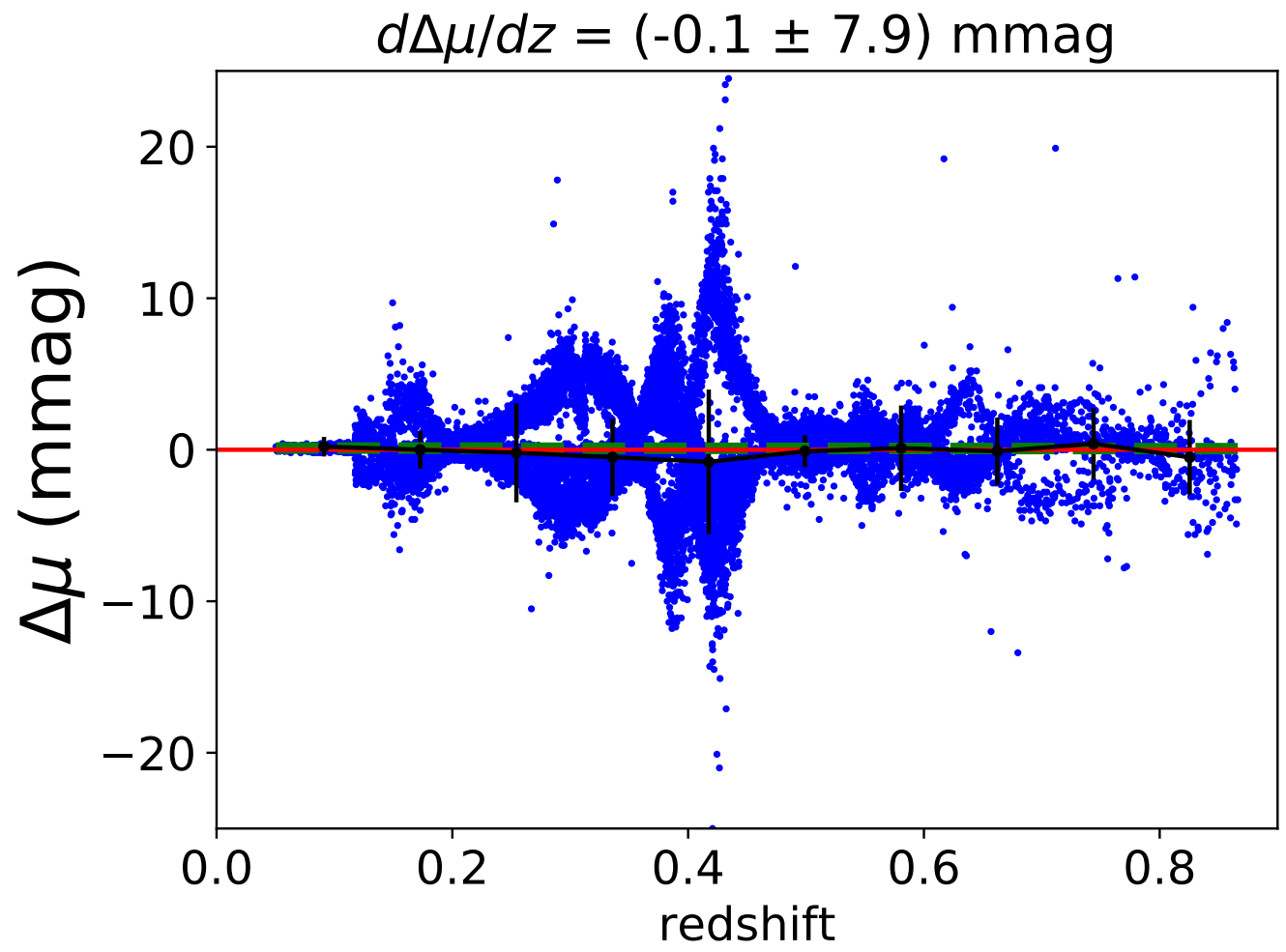

Figure 10. Same as Fig. 9, but for instrumental corrections only. 

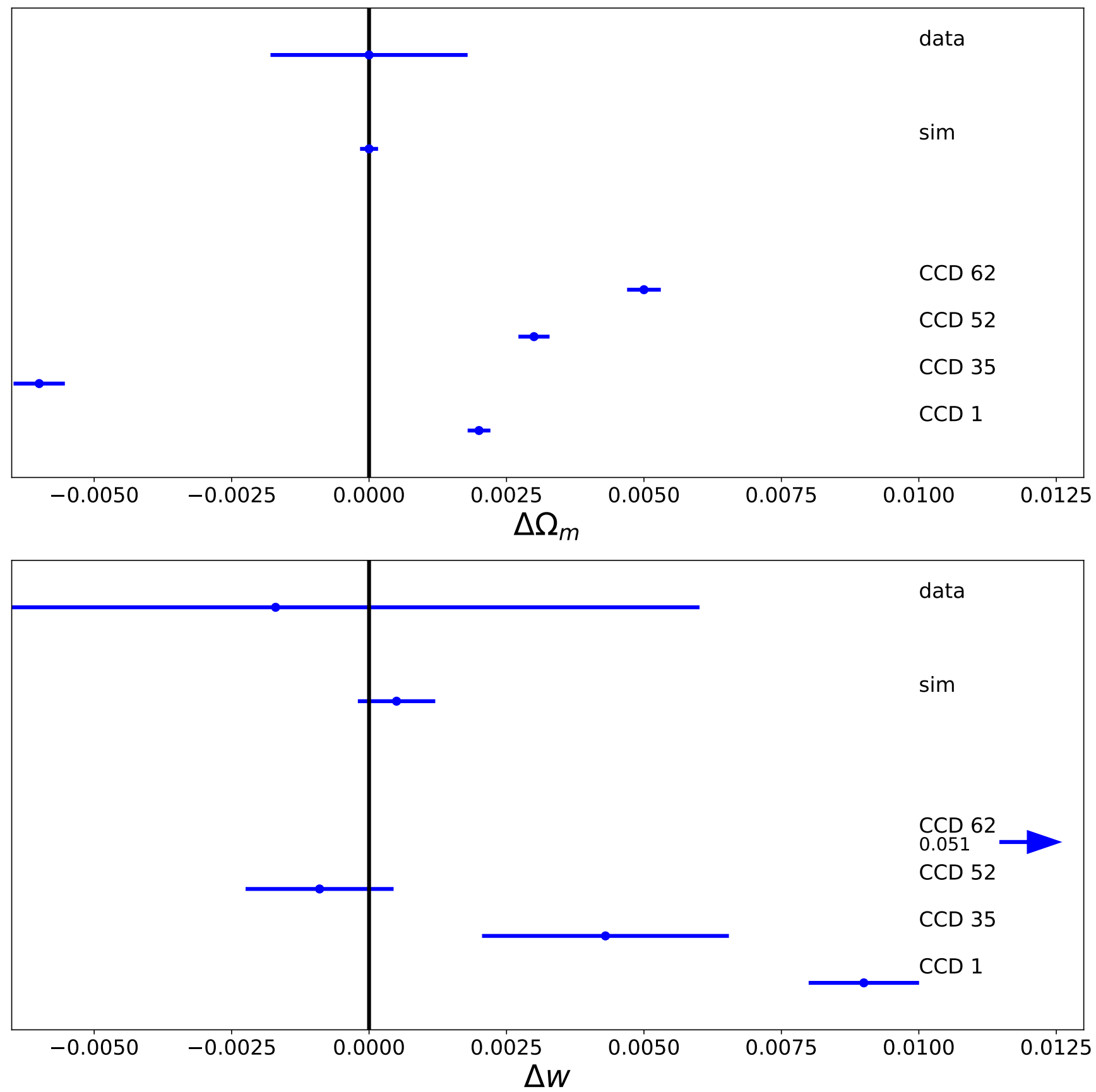

Figure 11. $\Delta \Omega_{m}$ and $\Delta w$ (Equations 9 and 10) for the labeled samples. $\Delta w$ for CCD62 is out of view so its value is printed next to an arrow.

Science Institute, which is operated by the Association of Universities for Research in Astronomy, Inc., for NASA, under contract NAS 5-26555.

This paper makes use of observations taken using the AngloAustralian Telescope under programs ATAC A/2013B/12 and NOAO 2013B-0317; the Gemini Observatory under programs NOAO 2013A-0373/GS-2013B-Q-45, NOAO 2015B-0197/GS2015B-Q-7, and GS-2015B-Q-8; the Gran Telescopio Canarias under programs GTC77-13B, GTC70-14B, and GTC101-15B; the Keck Observatory under programs U063-2013B, U021-2014B, U048-2015B, U038-2016A; the Magellan Observatory under pro- grams CN2015B-89; the MMT under 2014c-SAO-4, 2015a-SAO12, 2015c-SAO-21; the South African Large Telescope under programs 2013-1-RSA_OTH-023, 2013-2-RSA_OTH-018, 20141-RSA_OTH-016, 2014-2-SCI-070, 2015-1-SCI-063, and 20152-SCI-061; and the Very Large Telescope under programs ESO 093.A-0749(A), 094.A-0310(B), 095.A-0316(A), 096.A-0536(A), 095.D-0797(A).

Funding for the DES Projects has been provided by the U.S. Department of Energy, the U.S. National Science Foundation, the Ministry of Science and Education of Spain, the Science and Technology Facilities Council of the United Kingdom, the 

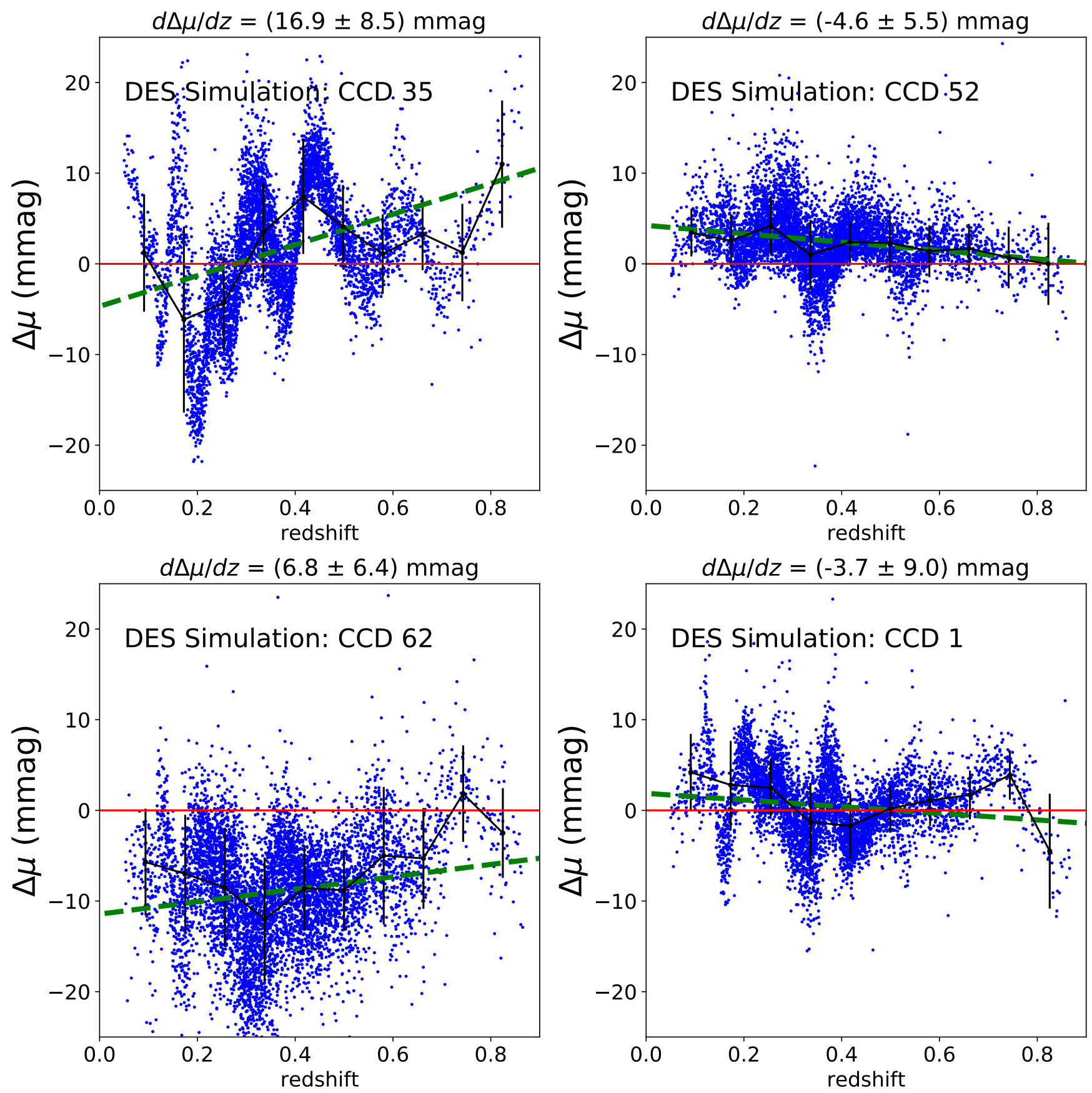

Figure 12. For simulated DES-like supernova, $\Delta \mu$ (Equation 5) vs. redshift for the labeled CCDs.

Higher Education Funding Council for England, the National Center for Supercomputing Applications at the University of Illinois at Urbana-Champaign, the Kavli Institute of Cosmological Physics at the University of Chicago, the Center for Cosmology and AstroParticle Physics at the Ohio State University, the Mitchell Institute for Fundamental Physics and Astronomy at Texas A\&M University, Financiadora de Estudos e Projetos, Fundação Carlos Chagas Filho de Amparo à Pesquisa do Estado do Rio de Janeiro, Conselho Nacional de Desenvolvimento Científico e Tecnológico and the Ministério da Ciência, Tecnologia e Inovação, the Deutsche
Forschungsgemeinschaft and the Collaborating Institutions in the Dark Energy Survey.

The Collaborating Institutions are Argonne National Laboratory, the University of California at Santa Cruz, the University of Cambridge, Centro de Investigaciones Energéticas, Medioambientales y Tecnológicas-Madrid, the University of Chicago, University College London, the DES-Brazil Consortium, the University of Edinburgh, the Eidgenössische Technische Hochschule (ETH) Zürich, Fermi National Accelerator Laboratory, the University of Illinois at Urbana-Champaign, the Institut de Ciències de l'Espai (IEEC/CSIC), the Institut de Física d'Altes Energies, Lawrence 
Berkeley National Laboratory, the Ludwig-Maximilians Universität München and the associated Excellence Cluster Universe, the University of Michigan, the National Optical Astronomy Observatory, the University of Nottingham, The Ohio State University, the University of Pennsylvania, the University of Portsmouth, SLAC National Accelerator Laboratory, Stanford University, the University of Sussex, Texas A\&M University, and the OzDES Membership Consortium.

Based in part on observations at Cerro Tololo Inter-American Observatory, National Optical Astronomy Observatory, which is operated by the Association of Universities for Research in Astronomy (AURA) under a cooperative agreement with the National Science Foundation.

The DES data management system is supported by the National Science Foundation under Grant Numbers AST-1138766 and AST-1536171. The DES participants from Spanish institutions are partially supported by MINECO under grants AYA201571825, ESP2015-66861, FPA2015-68048, SEV-2016-0588, SEV2016-0597, and MDM-2015-0509, some of which include ERDF funds from the European Union. IFAE is partially funded by the CERCA program of the Generalitat de Catalunya. Research leading to these results has received funding from the European Research Council under the European Union's Seventh Framework Program (FP7/2007-2013) including ERC grant agreements 240672, 291329, and 306478. We acknowledge support from the Australian Research Council Centre of Excellence for All-sky Astrophysics (CAASTRO), through project number CE110001020, and the Brazilian Instituto Nacional de Ciência e Tecnologia (INCT) e-Universe (CNPq grant 465376/2014-2).

This manuscript has been authored by Fermi Research Alliance, LLC under Contract No. DE-AC02-07CH11359 with the U.S. Department of Energy, Office of Science, Office of High Energy Physics. The United States Government retains and the publisher, by accepting the article for publication, acknowledges that the United States Government retains a non-exclusive, paid-up, irrevocable, world-wide license to publish or reproduce the published form of this manuscript, or allow others to do so, for United States Government purposes.

\section{REFERENCES}

Astier P., Guy J., Regnault N., et al., 2006, A\&A, 447, 31

Berk A., Bernstein L. S., Robertson D. C., 1987, Technical report, MODTRAN: A moderate resolution model for LOWTRAN

Bernstein J. P., Kessler R., Kuhlmann S., et al., 2012, ApJ, 753, 152

Betoule M., Kessler R., Guy J., et al., 2014, A\&A, 568, A22

Blake C. H., Shaw M. M., 2011, PASP, 123, 1302

Bohlin R. C., Gordon K. D., Tremblay P.-E., 2014, PASP, 126, 711

Brout D., Scolnic D., Kessler R., et al., 2018b, arXiv e-prints,

Brout D., Sako M., Scolnic D., et al., 2018a

Burke D. L., Rykoff E. S., Allam S., et al., 2018, AJ, 155, 41

Conley A., Guy J., Sullivan M., et al., 2011, ApJS, 192, 1

D'Andrea C., Smith M., Sullivan M., et al., 2018, preprint (arXiv: 1811.09565)

DES Collaboration 2018, preprint (arXiv: 1811.02374)

Diehl H. T., Neilsen E. Gruendl R. A., et al., 2018, Dark energy survey operations: years 4 and 5, doi:10.1117/12.2312113, https://doi.org/10.1117/12.2312113

Drlica-Wagner A., Sevilla-Noarbe I., Rykoff E. S., et al., 2018, ApJS, 235,33

Eisenstein D. J., Zehavi I., Hogg D. W., et al., 2005, ApJ, 633, 560

Finkbeiner D. P., Schlafly E. F., Schlegel D. J., et al., 2016, ApJ, 822, 66

Flaugher B., Diehl H. T., Honscheid K., et al., 2015, AJ, 150, 150

Fukugita M., Ichikawa T., Gunn J. E., et al., 1996, AJ, 111, 1748
Gupta R. R., Kuhlmann S., Kovacs E., et al., 2016, AJ, 152, 154

Guy J., Astier P., Baumont S., et al., 2007, A\&A, 466, 11

Guy J., Sullivan M., Conley A., et al., 2010, A\&A, 523, A7

Hicken M., Challis P., Jha S., et al., 2009, ApJ, 700, 331

Hicken M., Challis P., Kirshner R. P., et al., 2012, ApJS, 200, 12

Holtzman J. A., Marriner J., Kessler R., et al., 2008, AJ, 136, 2306

Hounsell R., Scolnic D., Foley R. J., et al., 2017, preprint, (arXiv: 1702.01747)

Hsiao E. Y., Conley A., Howell D. A., et al., 2007, ApJ, 663, 1187

Ivezić Ž.., the LSST Science Collaboration 2013, LSST Science Requirements Document, http://1s.st/LPM- 17

James F., Roos M., 1975, Computer Physics Communications, 10, 343

Kessler R., Scolnic D., 2017, ApJ, 836, 56

Kessler R., Bernstein J. P., Cinabro D., 2009, PASP, 121, 1028

Kessler R., Guy J., Marriner J., et al., 2013, ApJ, 764, 48

Kessler R., Marriner J., Childress M., et al., 2015, AJ, 150, 172

Kessler R., Asorey J., Brout D., et al., 2018

Komatsu E., Dunkley J., Nolta M. R., et al., 2009, ApJS, 180, 330

Li T. S., DePoy D. L., Marshall J. L., et al., 2016, AJ, 151, 157

Magnier E. A., Schlafly E., Finkbeiner D., et al., 2013, ApJS, 205, 20

Marshall J. L., Rheault J.-P., DePoy D. L., et al., 2013, preprint, (arXiv: 1302.5720 )

Mosher J., Guy J., Kessler R., et al., 2014, ApJ, 793, 16

Oke J. B., Gunn J. E., 1983, ApJ, 266, 713

Padmanabhan N., Schlegel D. J., Finkbeiner D. P., et al., 2008, ApJ, 674,1217

Perrett K., Sullivan M., Conley A., et al., 2012, AJ, 144, 59

Regnault N., Conley A., Guy J., et al., 2009, A\&A, 506, 999

Rest A., Scolnic D., Foley R. J., et al., 2014, ApJ, 795, 44

Riess A. G., Strolger L.-G., Tonry J., et al., 2004, ApJ, 607, 665

Riess A. G., Strolger L.-G., Casertano S., et al., 2007, ApJ, 659, 98

Rodney S. A., Riess A. G., Strolger L.-G., et al., 2014, AJ, 148, 13

Sako M., Bassett B., Becker A. C., et al., 2014, preprint, (arXiv: 1401.3317)

Schlafly E. F., Finkbeiner D. P., Jurić M., et al., 2012, ApJ, 756, 158

Scolnic D., Kessler R., 2016, ApJ, 822, L35

Scolnic D., Casertano S., Riess A., et al., 2015, ApJ, 815, 117

Scolnic D. M., Jones D. O., Rest A., 2017, preprint, (arXiv: 1710.00845)

Stritzinger M. D., Phillips M. M., Boldt L. N., et al., 2011, AJ, 142, 156

Suzuki N., Rubin D., Lidman C., et al., 2012, ApJ, 746, 85

The Dark Energy Survey Collaboration 2005, ArXiv Astrophysics e-prints,

Tonry J. L., Stubbs C. W., Lykke K. R., et al., 2012, ApJ, 750, 99

Tripp R., 1998, A\&A, 331, 815

York D. G., Adelman J., Anderson Jr. J. E., et al., 2000, AJ, 120, 1579

\section{APPENDIX A: AUTHOR AFFILIATIONS}

1 Department of Astronomy and Astrophysics, University of Chicago, Chicago, IL 60637, USA

${ }^{2}$ Kavli Institute for Cosmological Physics, University of Chicago, Chicago, IL 60637, USA

${ }^{3}$ Department of Physics and Astronomy, University of Pennsylvania, Philadelphia, PA 19104, USA

${ }^{4}$ Kavli Institute for Particle Astrophysics \& Cosmology, P. O. Box 2450, Stanford University, Stanford, CA 94305, USA

5 SLAC National Accelerator Laboratory, Menlo Park, CA 94025, USA

${ }^{6}$ School of Mathematics and Physics, University of Queensland,

Brisbane, QLD 4072, Australia

7 Lawrence Berkeley National Laboratory, 1 Cyclotron Road, Berkeley, CA 94720, USA

${ }^{8}$ Fermi National Accelerator Laboratory, P. O. Box 500, Batavia, IL 60510, USA

${ }^{9}$ The Research School of Astronomy and Astrophysics, Australian National University, ACT 2601, Australia 
10 Institute of Cosmology and Gravitation, University of Portsmouth, Portsmouth, PO1 3FX, UK

11 ARC Centre of Excellence for All-sky Astrophysics (CAASTRO)

12 School of Physics and Astronomy, University of Southampton, Southampton, SO17 1BJ, UK

13 African Institute for Mathematical Sciences, 6 Melrose Road, Muizenberg, 7945, South Africa

${ }^{14}$ South African Astronomical Observatory, P.O.Box 9, Observatory 7935, South Africa

15 Cerro Tololo Inter-American Observatory, National Optical Astronomy Observatory, Casilla 603, La Serena, Chile

${ }^{16}$ LSST, 933 North Cherry Avenue, Tucson, AZ 85721, USA

17 CNRS, UMR 7095, Institut d'Astrophysique de Paris, F-75014, Paris, France

18 Sorbonne Universités, UPMC Univ Paris 06, UMR 7095, Institut d'Astrophysique de Paris, F-75014, Paris, France

19 Department of Physics \& Astronomy, University College London, Gower Street, London, WC1E 6BT, UK

20 Centro de Investigaciones Energéticas, Medioambientales y Tecnológicas (CIEMAT), Madrid, Spain

21 Laboratório Interinstitucional de e-Astronomia - LIneA, Rua Gal. José Cristino 77, Rio de Janeiro, RJ - 20921-400, Brazil

22 Department of Astronomy, University of Illinois at UrbanaChampaign, 1002 W. Green Street, Urbana, IL 61801, USA

23 National Center for Supercomputing Applications, 1205 West Clark St., Urbana, IL 61801, USA

${ }^{24}$ Institut de Física d'Altes Energies (IFAE), The Barcelona Institute of Science and Technology, Campus UAB, 08193 Bellaterra (Barcelona) Spain

25 Institut d'Estudis Espacials de Catalunya (IEEC), 08034 Barcelona, Spain

${ }^{26}$ Institute of Space Sciences (ICE, CSIC), Campus UAB, Carrer de Can Magrans, s/n, 08193 Barcelona, Spain

27 INAF, Astrophysical Observatory of Turin, I-10025 Pino Torinese, Italy

28 Observatório Nacional, Rua Gal. José Cristino 77, Rio de Janeiro, RJ - 20921-400, Brazil

${ }^{29}$ Instituto de Fisica Teorica UAM/CSIC, Universidad Autonoma de Madrid, 28049 Madrid, Spain

${ }^{30}$ Santa Cruz Institute for Particle Physics, Santa Cruz, CA 95064, USA

31 Center for Cosmology and Astro-Particle Physics, The Ohio State University, Columbus, OH 43210, USA

32 Department of Physics, The Ohio State University, Columbus, OH 43210, USA

${ }^{33}$ Harvard-Smithsonian Center for Astrophysics, Cambridge, MA 02138, USA

34 Department of Astronomy/Steward Observatory, 933 North Cherry Avenue, Tucson, AZ 85721-0065, USA

35 Australian Astronomical Optics, Macquarie University, North Ryde, NSW 2113, Australia

${ }^{36}$ Departamento de Física Matemática, Instituto de Física, Universidade de São Paulo, CP 66318, São Paulo, SP, 05314-970, Brazil

37 George P. and Cynthia Woods Mitchell Institute for Fundamental Physics and Astronomy, and Department of Physics and Astronomy, Texas A\&M University, College Station, TX 77843, USA

38 Department of Astronomy, The Ohio State University, Columbus, OH 43210, USA

39 Department of Astronomy, University of Michigan, Ann Arbor, MI 48109, USA
40 Department of Physics, University of Michigan, Ann Arbor, MI 48109, USA

41 Institució Catalana de Recerca i Estudis Avançats, E-08010 Barcelona, Spain

42 Jet Propulsion Laboratory, California Institute of Technology, 4800 Oak Grove Dr., Pasadena, CA 91109, USA

43 Brandeis University, Physics Department, 415 South Street, Waltham MA 02453

44 Instituto de Física Gleb Wataghin, Universidade Estadual de Campinas, 13083-859, Campinas, SP, Brazil

45 Computer Science and Mathematics Division, Oak Ridge National Laboratory, Oak Ridge, TN 37831 